\title{
1 Septins coordinate cell wall integrity and lipid metabolism in 2 a sphingolipid-dependent process
}

3

$4 \quad$ Alexander Mela ${ }^{1+}$ and Michelle Momany ${ }^{1^{*}}$

5

6

7

$8 \quad{ }^{1}$ Fungal Biology Group and Plant Biology Department, University of Georgia,

92502 Miller Plant Science Building, Athens, GA 30602

10

11

$12{ }^{*}$ Corresponding Author:

13 Email: mmomany@uga.edu (MM)

$14+$ Current address:

15 Department of Plant \& Microbial Biology, University of California, Berkeley

16 Keywords: Septins; MAPK Signaling; Cell wall integrity; Sterol Rich Domains

17 
During normal development and response to environmental stress, fungi must coordinate synthesis of the cell wall and plasma membrane. Septins, small cytoskeletal GTPases, colocalize with membrane sterol-

21 rich regions and facilitate recruitment of cell wall synthases during dynamic wall remodeling. In this

22 study we show that null mutants missing an Aspergillus nidulans core septin present in hexamers and

23 octamers $\left(\Delta a s p A^{c d c 11}, \Delta a s p B^{c d c 3}\right.$, or $\left.\Delta a s p C^{c d c 12}\right)$ are sensitive to multiple cell wall-disturbing agents

24 known to activate the cell wall integrity MAPK pathway and that this sensitivity can be remediated by osmotic support. The null mutant missing the octamer-exclusive core septin $\left(\Delta a s p D^{\text {cdc10 }}\right)$ showed similar osmotic-remedial sensitivity, but only to a single cell wall-disturbing agent and the null mutant missing the noncore septin ( $\triangle a s p E$ ) showed very mild osmotic-remedial sensitivity to a different single agent. Representative core septin null mutants showed changes in cell wall polysaccharide composition, organization, and chitin synthase localization. Double mutant analysis with $\Delta m p k A$ suggested core septins interact with the cell wall integrity pathway. Null mutants missing any of the five septins were resistant to ergosterol-disrupting agents. The $\Delta a s p A^{c d c 11}, \Delta a s p B^{c d c 3}$, and $\Delta a s p C^{c d c 12}$ mutants showed increased sensitivity to sphingolipid-disrupting agents that was remediated by addition of exogenous

33 phytosphingosine. Representative core septins were mislocalized after treatment with sphingolipiddisrupting agents, but not after treatment with ergosterol-disrupting agents. When challenged with both sphingolipid-disturbing and cell wall-disturbing agents in combination, remediation of the lipid defect restored proper growth to $\Delta a s p A^{c d c 11}, \Delta a s p B^{c d c 3}$, and $\Delta a s p C^{c d c 12}$, but remediation of the cell wall defect did

37 not. Our data suggest that the core hexamer and octamer septins are involved in cell wall integrity signaling with the noncore septin playing a minor role; that all five septins are involved in monitoring 
43 The cell wall and plasma membrane (PM) are the primary lines of defense against environmental insults

44 for fungal cells. With the large surface area of hyphal networks and intimate contact with the surrounding

45 media, fungi encounter many stresses, ranging from ion imbalance to predation. The cell wall contains

46 several polysaccharide constituents: chitin provides the rigid framework of the cell wall, $\beta$-glucan

47 maintains the shape, and mannans form the outermost, protective layer (1-6). Precise regulation of cell

48 wall and plasma membrane architecture is attained by tightly coordinated signaling pathways which

49 control genes responsible for maintaining homeostasis between the intracellular and extracellular

50 environments.

51 Septins are highly conserved small GTPase cytoskeletal proteins that function as molecular scaffolds for

52 dynamic cell wall and plasma membrane-remodeling, as well as diffusion barriers restricting movement

53 of membrane and cell wall-associated molecules (7-14). The Saccharomyces cerevisiae septins Cdc3,

54 Cdc10, Cdc11, and Cdc12 have been termed 'core septins' because they are monomers which assemble

55 into non-polar heterooligomers and micrometer-scale higher order structures in the form of bars, rings, or

56 gauzes (15-18). A. nidulans contains four orthologous septin proteins: AspA ${ }^{\text {Cdc11, AspB }}{ }^{\text {Cdc3 }}$, AspC ${ }^{\text {Cdc12 }}$,

57 and AspD ${ }^{\mathrm{Cdc10}}$, as well as AspE, which has no S. cerevisiae orthologue (19). A. nidulans contains two

58 distinct subpopulations of heterooligomers; an octameric oligomer consisting of all four core septins in

59 the same order as proposed in S. cerevisiae (AspA ${ }^{\text {Cdc11 }}$-AspC ${ }^{\text {Cdc12 }-A s p B}{ }^{\text {Cdc3 }}$ - AspD ${ }^{\text {Cdc10 }}-$ AspD $^{\text {Cdc10 }}$-AspB

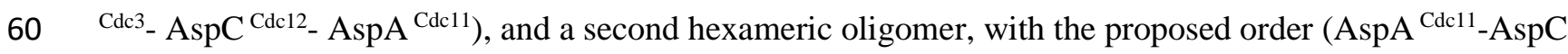

61 Cdc12 $-A s p B^{\text {Cdc3 }-A s p B}{ }^{\text {Cdc3 }-A s p C ~}{ }^{\text {Cdc12 }}$ AspA $\left.{ }^{\text {Cdc11 }}\right)(12,13,20)$. For clarity, we will refer to AspA ${ }^{\text {Cdc11, }}$,

$62 \mathrm{AspB}^{\mathrm{Cdc3}}$, and AspC ${ }^{\text {Cdc12 }}$ as 'core hexamer septins'; AspA ${ }^{\mathrm{Cdc11}}, \mathrm{AspB}^{\mathrm{Cdc3}}, \mathrm{AspC}^{\text {Cdc12 }}$, and AspD ${ }^{\text {Cdc10 as }}$

63 'core octamer septins'; and AspE as the 'noncore septin' because it does not assemble into oligomeric

64 structures, though it is required for higher order structure assembly at the multicellular stage (20, 21). 
Previous studies in Candida albicans have shown that septins provide the scaffolding for cell wall proteins via the Cell Wall Integrity (CWI) MAPK signaling pathway (7, 9, 22-24). The cell wall

67 integrity pathway, along with the other major MAPK signaling pathways (High Osmolarity Glycerol (HOG), cAMP-PKA, Target of Rapamycin (TOR), Calcineurin/Calcium, and Mating/Pheromone response pathways) are highly conserved across the Kingdom Fungi, and have been shown to undergo extensive cross-talk to coordinate virtually all biological functions in the cell, from expansion and division to asexual reproduction (25-32). Sphingolipids are long chain base-containing lipids (33) that are metabolized in a highly conserved pathway in plants, animals, and fungi; sphingolipid metabolism shares direct connections to other major metabolic pathways, such as sterol metabolism and fatty acid and phospholipid synthesis (34). Sphingolipid pathway intermediates, such as phytosphingosine, have been shown to be involved in CWI pathway signaling in S. cerevisiae (33, 35). Sterol rich domains (SRDs), also called 'lipid rafts' or 'lipid microdomains,' are regions of the plasma membrane enriched in specific classes of lipids, including sterols (ergosterol, the major sterol found in most fungi (36)), sphingolipids, and phosphoinositides (37,

79 38), which have been shown to be functionally important for maintenance of cell polarity (39).

80 Membrane organization, plasticity, and overall integrity have been attributed to sphingolipid, sterol, and

81 glycerophospholipid interactions (40). In vitro work has shown that yeast septins bind directly to the

82 phosphoinositides PIP2, PI(4,5)P2, PI(5)P, and PI(4)P (41-44). Septins have also been shown to genetically interact with Sur2, a sphinganine C4-hydroxylase which catalyzes the conversion of

84 sphinganine to phytosphingosine in sphingolipid biosynthesis (45), and to physically interact with

85 GTPases, Bud1 and Cdc42 to maintain sphingolipid-dependent diffusion barriers at cell membranes in yeast (46). Interdependent colocalization of septins and sterol rich microdomains have been described in

87 a number of in vivo and in vitro systems $(47,48)$. In the dimorphic fungus $U$. maydis, septins and sterol

88 rich domains depend on each other to localize properly at the hyphal tip for cell signaling and cell polarity establishment (49). In the budding yeast $S$. cerevisiae, long-chain sphingolipids have been implicated in 
maintaining asymmetric distribution and mobility of some membrane-spanning molecules, such as

91 multidrug resistance transporters (50). In contrast to these findings, a more recent comprehensive study

92 of protein segregation during cell division in budding yeast found that the majority of ER and PM

93 proteins with transmembrane domains were actually symmetrically segregated, however septins were

94 shown to be responsible for partitioning of the PM-associated ER at the bud neck, thereby restricting

95 diffusion of ER lumen and a particular set of membrane-localized proteins (51).

96

97

Recent work has started to unravel the functional connections between the septins, cell wall integrity MAPK pathway signaling, and lipid metabolism, however most studies have focused on a small sub-set of septin monomers and/or were conducted in primarily yeast-type fungi (52-57). Here we show in the filamentous fungus $A$. nidulans that the core septins, $A s p A^{\mathrm{Cdc11}}, \mathrm{AspB}^{\mathrm{Cdc3}}, \mathrm{AspC}^{\mathrm{Cdc12}}$, and $\mathrm{AspD}^{\mathrm{Cdc10}}$ are required for proper coordination of the cell wall integrity pathway, that all five septins are involved in lipid metabolism, and that these roles require sphingolipids.

\section{Results}

Mutants missing core hexamer septins are hypersensitive to multiple cell wall-disturbing agents. The mutant missing the octamer-exclusive core septin $A s p D^{C d c 10}$ is sensitive to a single agent and the mutant missing the noncore septin AspE is mildly sensitive to a single agent. To determine whether $A$. nidulans septins are important for cell wall integrity, we used spore dilution assays to test the growth of septin deletion mutants on media containing a variety of known cell wall-disturbing agents. Wild type and septin null mutants were tested on Calcofluor White (CFW) and Congo Red (CR), cell wall polymerintercalating agents that perturb chitin and $\beta$-1,3-glucan, respectively; Caspofungin (CASP), an inhibitor of $\beta$-1,3-glucan synthase; and Fludioxonil (FLU), a phenylpyrrol fungicide that antagonizes the group III histidine kinase in the osmosensing pathway and consequently affects cell wall integrity pathway signaling (Fig 1)(58-67). Septin mutants $\Delta a s p A^{c d c 11}, \Delta a s p B^{c d c 3}$, and $\Delta a s p C^{c d c 12}$ showed hypersensitivity to CFW, CR, CASP, and FLU; $\Delta a s p D^{c d c 10}$ displayed no sensitivity to CFW, CR, and FLU, and sensitivity to 
CFW, CASP, and FLU, and very mild sensitivity to CR. (Fig 1A).

117 hallmark of cell wall integrity defects is rescue by the addition of an osmotic stabilizer, such as sorbitol or

(Fig 1B). Sorbitol also remediated the sensitivity of $\Delta a s p D^{c d c 10}$ to CASP and the mild sensitivity of

A
A

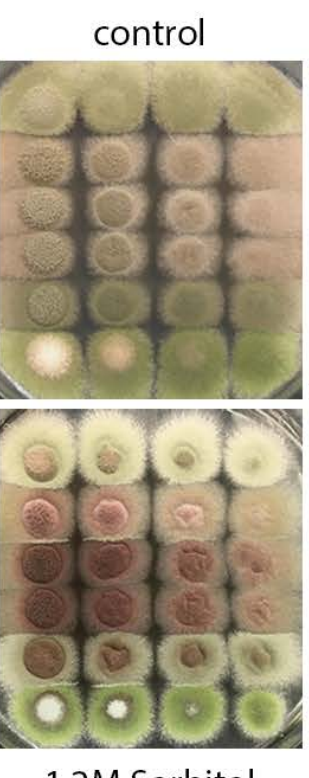

1.2M Sorbitol
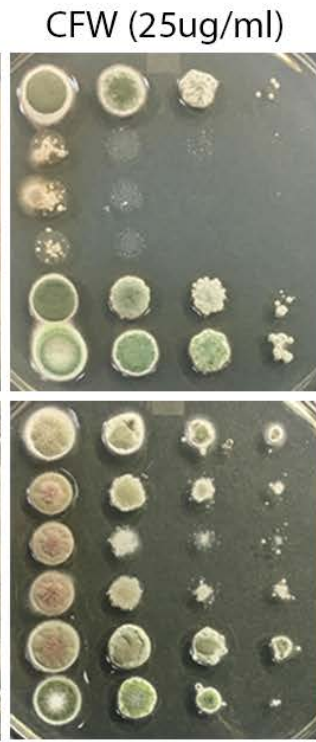

CFW $(25 \mathrm{ug} / \mathrm{ml})$

$+$ 1.2M Sorbitol

Fig 1. Septin null mutants exhibit sensitivity to cell wall-disturbing agents, which can be remediated by osmotic support. (Top Row) Solid media spotting assay. WT and septin null mutants $\Delta a s p A^{\text {cdc11 }}$, $\Delta a s p B^{c d c 3}, \Delta a s p C^{c d c 12}, \Delta a s p D^{c d c 10}$, and $\Delta a s p E$ were tested for sensitivity by spotting decreasing spore concentrations on complete medium plates with or without cell wall-disturbing agents Calcofluor White (CFW), Congo Red (CR), Caspofungin (CASP), and Fludioxonil (FLU) at the indicated final concentrations. (Bottom Row) WT and septin null mutants were tested for osmotic remediation of hypersensitivity to cell wall-disturbing agents, by spotting decreasing spore concentrations on media 
amended with exogenous $1.2 \mathrm{M}$ sorbitol. Spore concentrations were $10^{7} \mathrm{conidia} / \mathrm{mL}-10^{4} \mathrm{conidia} / \mathrm{mL}$ for all assays. Differences in colony color result from changes in spore production, spore pigment, and production of secondary metabolites under stress. $\mathrm{N} \geq 5$

\section{Core septin null mutant $\triangle a s p B^{c d c 3}$ has altered chitin and $\beta$-1,3-glucan localization. Because the core} septin mutants showed sensitivity to cell wall-disturbing agents consistent with action via the cell wall integrity pathway, we predicted that there might be differences in cell wall polymer localization in the mutants. To examine cell wall polymer localization, we did live-cell imaging of WT and $\Delta a s p B^{c d c 3}$ since it is a member of both hexamers and octamers. Conidia were incubated on coverslips in liquid media, stained with CFW or aniline blue to observe chitin and $\beta$-1,3-glucan, respectively, and immediately observed by fluorescence microscopy. Z-stack images were analyzed one-by-one, compressed into maximum projection images to visualize the fluorescence signal in the entire hyphal structure, and by line scans of aniline blue and calcofluor white staining patterns (Fig 2, S1 Fig). As previously reported, $\Delta a s p B^{c d c 3}$ showed more presumptive branch initials per hyphal compartment than WT (21). The aniline blue staining showed a reduction of labeling at all $\Delta a s p B^{c d c 3}$ hyphal tips. Smaller, less intense aniline blue labeling also occurred at presumptive branch initials (Fig 2A, S1A). The CFW staining of $\Delta a s p B^{c d c 3}$ showed a shift of the tip band of staining closer to the hyphal apex and a less well-defined endocytic collar zone (Fig 2B, S1B). These data show that cell wall organization is altered in $\Delta a s p B^{c d c 3}$ and raise the possibility that it might be altered in other core hexamer septin null mutants as well.

Core septin null mutant $\Delta a s p B^{c d c 3}$ has higher levels of chitin. Previous studies have shown that perturbations to one cell wall component often trigger compensatory changes to others via the cell wall integrity pathway $(28,68,69)$. To analyze the cell wall composition of septin mutants, two independent biological replicates of a diagnostic glycosyl linkage analysis (70) were conducted to quantify the cell wall polysaccharide composition of WT, $\Delta a s p B^{c d c 3}$ (a core septin null mutant which showed hypersensitivity to cell wall-disturbing agents), and $\Delta a s p E$ (non-core septin mutant)(S2 Fig). All samples contained 3- and 4-linked glucose as the primary cell wall components, as well as 4-linked Nacetylglucosamine (the monomer of chitin) and a relatively minor amount of mannan. The septin mutants 
A.
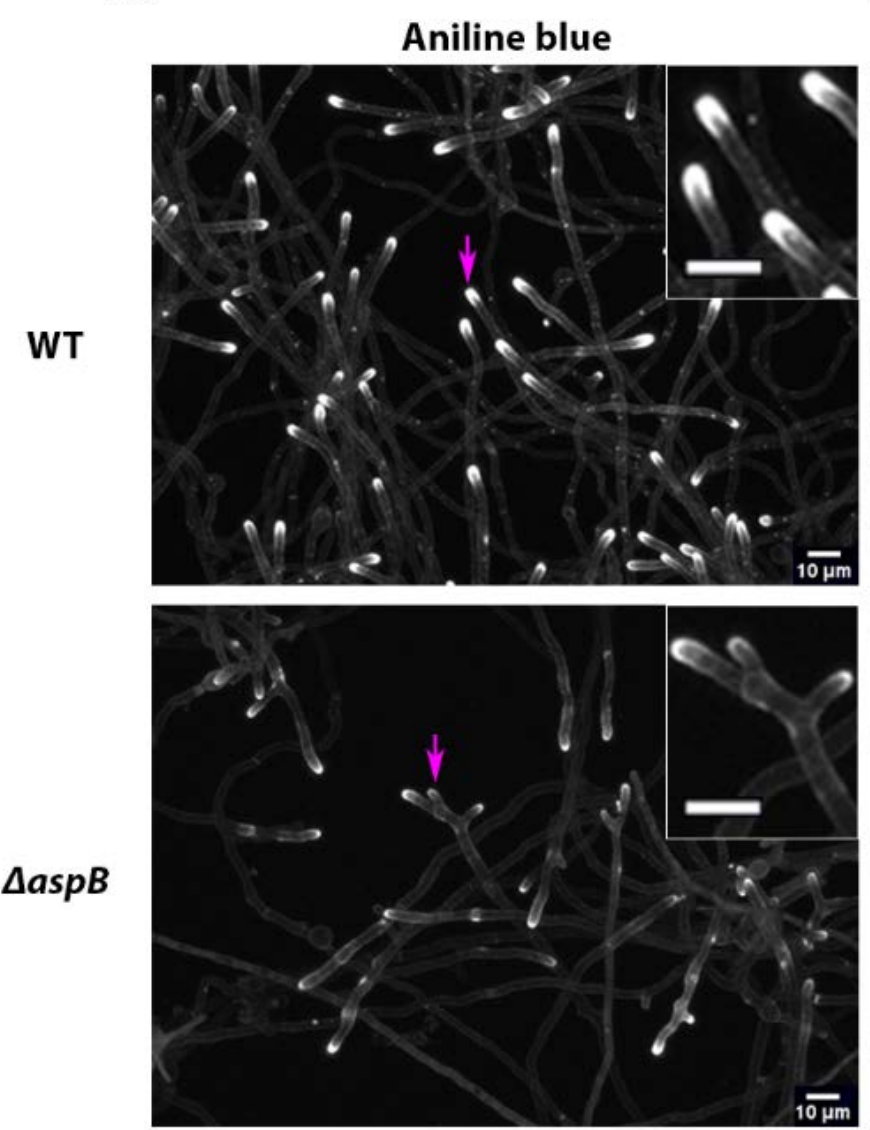

B.

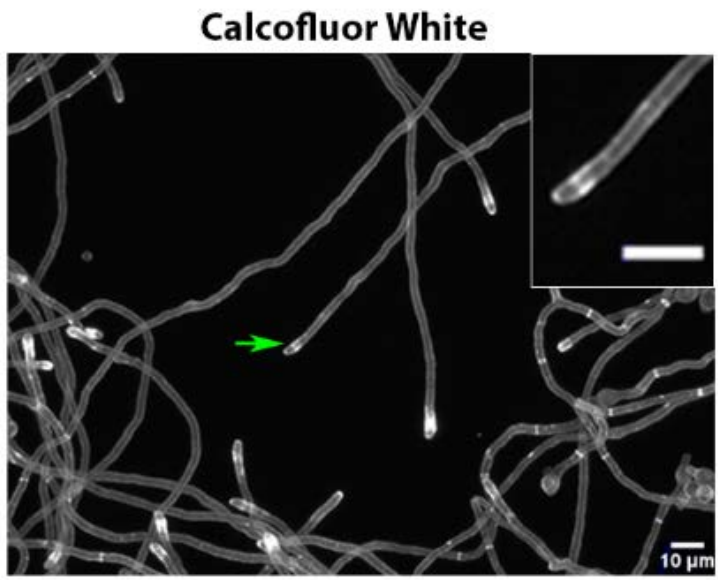

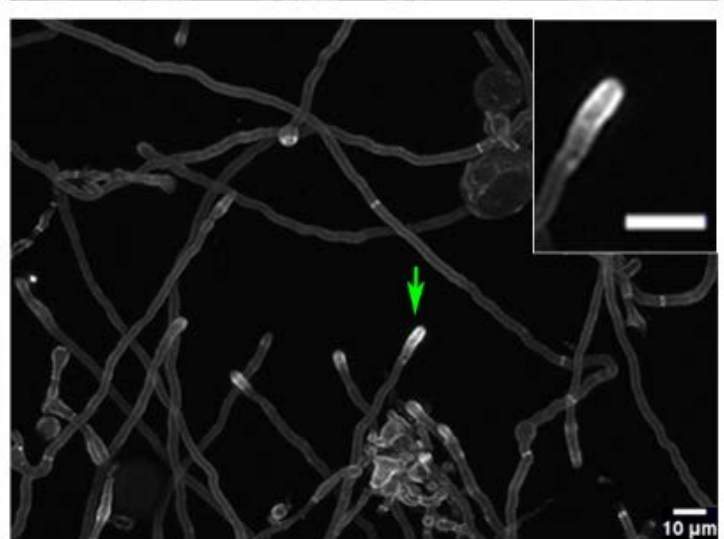

Fig 2. Cell wall staining reveals unique patterns of chitin and $\beta$-1,3-glucan deposition in the core septin hexamer null mutant $\Delta \boldsymbol{a s p B}^{c d c 3}$. (A) WT and $\Delta a s p B^{c d c 3}$ cells were incubated for approximately $14 \mathrm{~h}$ and (A) stained with aniline blue to visualize $\beta$-1,3-glucan or (B) stained with Calcofluor White (CFW) to visualize chitin. Representative images are shown from at least three independent biological replicates, with $\geq 100$ cells observed each. Magenta arrows in panel A denote hyphal tips with representative $\beta$-1,3-glucan deposition. Green arrows in panel B denote hyphal tips with representative chitin deposition. Insets show enlargement of area around the arrows. Scale bars $=10 \mu \mathrm{m}$. $\mathrm{N} \geq 3$ 
174 cell wall synthases are the ultimate effectors of the cell wall integrity pathway. A. nidulans contains six

175 genes for chitin synthases: $\operatorname{chs} A$, $\operatorname{chs} B, \operatorname{chs} C, \operatorname{chs} D, \operatorname{csm} A$, and $\operatorname{csm} B$. Chitin synthase B localizes to sites

176 of polarized growth in hyphal tips, as well as developing septa in vegetative hyphae and conidiophores, a

177 pattern very similar to septin localization. Deletion of chitin synthase B shows severe defects in most

178 filamentous fungi analyzed thus far, and repression of the chitin synthase B gene expression in chsA,

179 chs C, and chsD double mutants exacerbated growth defects from a number of developmental states

180 observed in each single mutant, suggesting it plays a major role in chitin synthesis at most growth stages

181 (22). For these reasons, we chose chitin synthase B as a candidate to observe in a septin mutant

background for possible defects in localization. We hypothesized that recruitment or maintenance of cell wall synthases, such as chitin synthase could be disrupted in septin null mutants which showed sensitivity to cell wall-disturbing agents and altered cell wall staining patterns. To determine the localization of synthases, a chitin synthase B-GFP (chsB-GFP) strain was crossed with strains in which core hexamer septins were deleted. After repeated attempts, the only successful cross was with core hexamer deletion strain $\triangle a s p A^{c d c 11}$. The $\triangle a s p A^{c d c 11}$, chsB-GFP strain showed conspicuous differences in chitin synthase localization patterns compared to WT (Fig 3 and Fig. S3). In WT, the chitin synthase-GFP signal was at the tips of $>90 \%$ of branches, presumptive branch initials, and apical hyphal tips (SFig 3A). In the septin null mutant $\Delta a s p A^{c d c 11}$, GFP signal was absent in most presumptive branch initials, but present in longer side branches that were at least $10 \mu \mathrm{m}$ long and in the apical hyphal tip(s) (Fig 3 and Fig. S3A). the increase in 4-GlcNAc levels seen in polysaccharide analysis (Fig. S3B). Calcofluor white labeling also showed that the polymer chitin was present throughout the hyphae in both the WT and septin deletion strains (Fig. 2) suggesting that chitin synthases had been active in both, but perhaps were not

196 retained as long in the presumptive branch initials of the septin deletion mutant. These data support the

197 hypothesis that core hexamer septins play a role in recruitment and/or retention of cell wall synthases to 198 the proper locations at the plasma membrane during growth and development. 


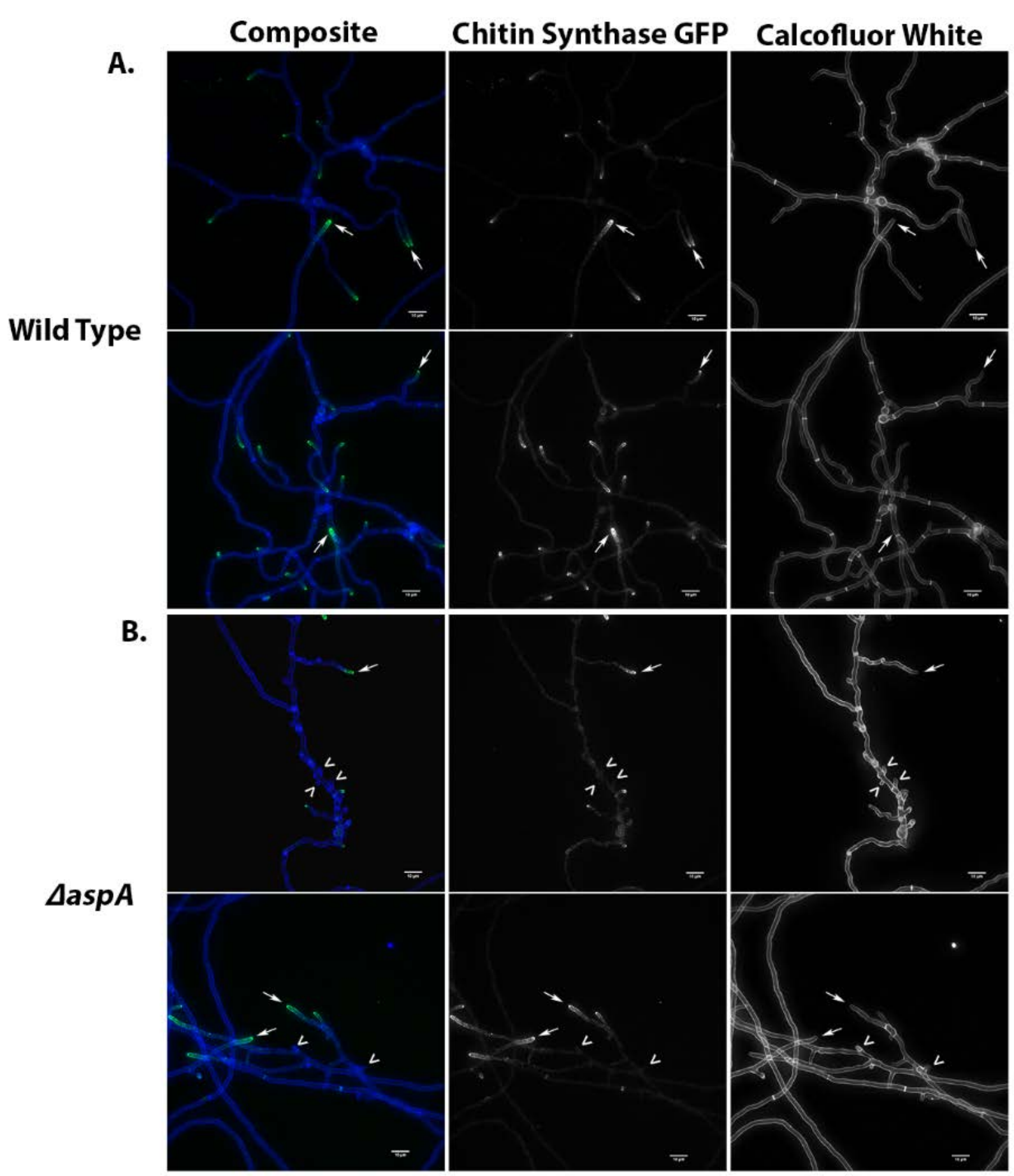

Fig 3. Chitin synthase is mislocalized in core septin null mutant $\Delta a s p A^{c d c 11}$. (A) Chitin synthase BGFP in WT background (Columns 1-2). (B) chitin synthase B-GFP in a $\Delta a s p A^{c d c 11}$ mutant background. Calcofluor White labeling shows the presence of the polymer chitin at septa, main hyphal tips, branches, and putative branch initials (Column 3). Representative images are shown from at least three independent biological replicates, with $\geq 100$ cells observed. White arrows highlight hyphal branches and tips. White arrow heads highlight putative branch initials. Scale bars $=10 \mu \mathrm{m} . \mathrm{N} \geq 3$. 
207 Double mutant analyses suggest the core septin $a s p B^{c d c 3}$ modulates the cell wall integrity pathway in

208 the $\Delta$ mpkA background under cell wall stress. To determine whether there are genetic interactions

209 between the septins and the cell wall integrity pathway kinases, double mutants were generated by sexual

210 crosses and analyzed. The first cell wall integrity pathway kinase, PkcA ${ }^{\mathrm{Pkc} 1}$ (ANID_00106), is essential in

211 A. nidulans, and so null alleles could not be utilized for double mutant analysis. A null allele of the final

212 kinase in the CWI MAPK signaling cascade, MpkA ${ }^{\text {Slt2 }}$ (ANID_11786), was crossed with $\Delta a s p B^{\text {cdc3 }}$ and

$213 \Delta a s p E$ and progeny were challenged with cell wall-disturbing treatments. If the septins are directly in the

214 CWI pathway, we would expect double mutants to show the same phenotypes as the parental null mutant

215 that acts earliest. If the septins are in a parallel pathway or alternate node which also affects cell wall

216 integrity, we would expect a novel/synergistic phenotype in the double mutants.

217 Spore dilution assays were conducted, challenging the double mutants and the parental strains with cell

218 wall-disturbing treatments. The double mutants $\Delta a s p B^{c d c 3} \Delta m p k A^{\text {slt2 }}$ and $\Delta a s p E \Delta m p k A^{\text {slt2 }}$ displayed a

219 colony-level radial growth defect and reduced conidiation which phenocopied the $\Delta m p k A^{\text {slt2 }}$ single

220 mutant. When challenged with low concentrations of CASP and CFW, the $\Delta a s p B^{\text {cdc3 }} \Delta m p k A^{\text {slt2 }}$ and

$221 \Delta a s p E \Delta m p k A^{\text {slt2 }}$ mutants were more sensitive than $\Delta a s p B^{c d c 3}$ and $\Delta a s p E$ single mutants, but suppressed

222 the colony growth defects of $\Delta m p k A^{\text {slt2 }}$. The novel phenotype of the double mutants shows that septins are

223 involved in cell wall integrity and raises the possibility that they act in a bypass or parallel node for

224 remediation of cell wall defects (Fig 4). 
A

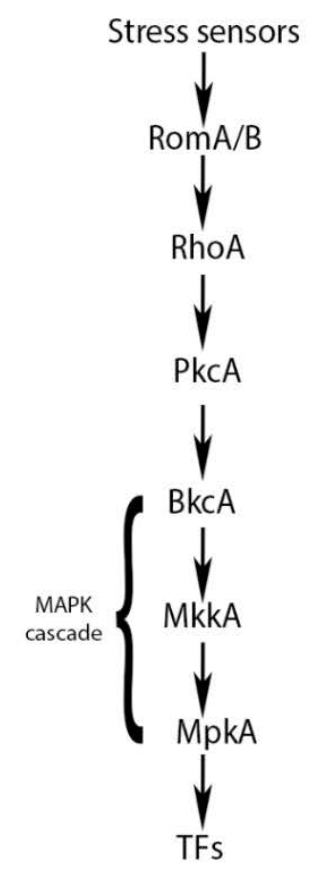

B
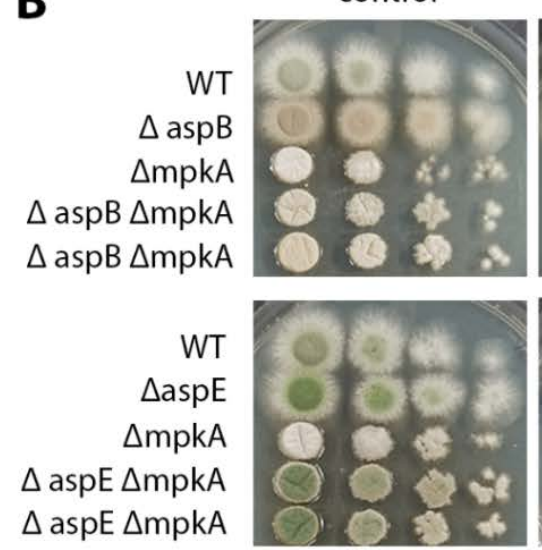
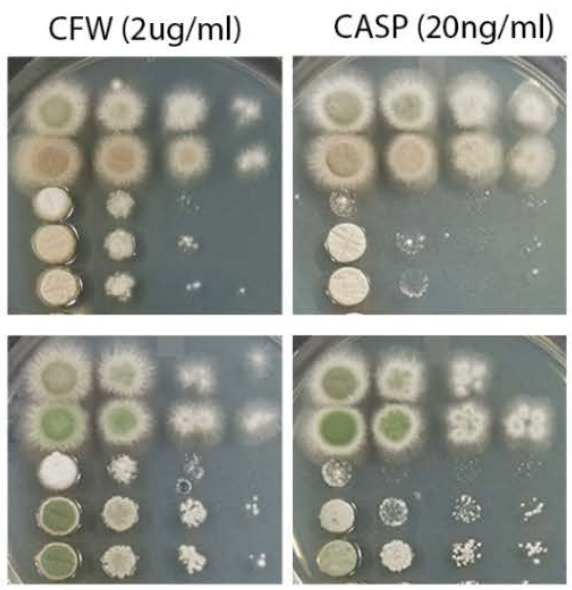

Fig 4. Double mutant analyses suggest core septins modulate the cell wall integrity pathway. (A) Simplified schematic diagram of the A. nidulans cell wall integrity MAPK signaling pathway (71). (B) Solid media spotting assays. (Top) WT, $\Delta a s p B^{c d c 3}, \Delta m p k A^{\text {slt2 }}$, and two $\Delta a s p B^{c d c 3} \Delta m p k A^{\text {slt2 }}$ double mutant strains, were tested for sensitivity by spotting decreasing spore concentrations on complete media plates with or without cell wall-disturbing agents. (Bottom) WT, $\Delta a s p E, \Delta m p k A^{\text {slt2 }}$, and two $\Delta a s p E \Delta m p k A^{\text {slt2 }}$ double mutant strains were tested for sensitivity by spotting decreasing spore concentrations on complete media plates with or without cell wall-disturbing agents. Differences in colony color result from changes in spore production, spore pigment, and production of secondary metabolites under stress. Transcription Factors, (TFs); Calcofluor White, (CFW); Caspofungin (CASP). Spore concentrations were $\left[10^{6}\right.$ conidia/mL $-10^{3}$ conidia/mL] for all assays in figure. $\mathrm{N}=3$

Core septin null mutants are insensitive to treatments which disrupt the Ca2+/Calcineurin, cAMP-

PKA, or TOR Pathways. Noncore septin null mutant $\triangle a s p E$ is sensitive to some TOR pathway

inhibitors. One possible explanation for the observed sensitivity to cell wall-disturbing agents could be that septins are involved in 'cross-talk' with other MAPK signaling pathways that have been shown to interact with cell wall integrity pathway signaling such as the Calcium/Calcineurin (CAMK) signaling pathway. To test this possibility, calcium chloride, EGTA (calcium chelating agent), and FK-506 
colony growth defects, suggesting that Ca2+/Calcineurin signaling pathway crosstalk does not significantly contribute to the observed sensitivity of septin null mutants to cell wall-disturbing agents.

Another pathway closely associated with cell wall integrity, lipid biosynthesis, and lipid signaling is the TOR MAPK signaling pathway (75). To test the involvement of septins in this signaling pathway, septin null mutants were challenged with rapamycin (a potent inhibitor of the TOR pathway), as well as methylxanthine derivatives and TOR pathway inhibitors, caffeine and theophylline (76-78). There were no observable growth defects in the presence of rapamycin (final concentration was $600 \mathrm{ng} / \mathrm{mL}$, which is approximately 3-fold higher than inhibitory concentration for known TOR pathway mutants) in the septin null mutants compared to WT (S4 Fig) $(79,80)$. Caffeine and theophylline have been shown to interfere with phosphodiesterase activity in the cAMP-PKA and TOR pathways, and $\Delta p k a A^{\text {tpk1 }}$ and $\Delta t o r 1^{\text {torA }}$ mutants show hypersensitivity to caffeine treatment which cannot be remediated by exogenous sorbitol $(58,78,81-83)$. If the septins were involved in crosstalk between the cAMP-PKA or TOR pathways and the CWI pathway, we would predict that septin null mutants would be hypersensitive to caffeine and theophylline treatments. To our surprise, only $\triangle a s p E$ showed hypersensitivity to both caffeine and theophylline, and the hypersensitivity was not remediated by an osmotic stabilizer (S4 Fig and data not shown). These data suggest that TOR, cAMP-PKA, and Calcium/Calcineurin signaling pathways do not contribute to the cell wall sensitivity or plasma membrane resistance phenotypes in the core septin null mutants, but the cAMP-PKA or TOR pathways may contribute to the phenotypes of the septin $\triangle a s p E$ null mutant.

\section{Core septin null mutants show increased resistance to disruption of ergosterol biosynthesis and $\Delta a s p A$} ${ }^{\text {cdc11 }}$ and $\triangle$ aspE show increased resistance to the ergosterol-binding drug Natamycin. We hypothesized that septins could indirectly modulate MAPK pathways, particularly the CWI pathway, through interactions with plasma membrane lipids within sterol rich domains (SRDs). Recent work has shown that the cell wall integrity pathway in S. cerevisiae is regulated by sphingolipids and ergosterol, facilitating proper deposition of cell wall polymers at actively growing regions and sites of septation (38, 
270 55). It is well-established that septins localize to sites of polarized growth and septation, where highly

271 dynamic remodeling of the plasma membrane and cell wall via membrane-bound synthases and polarity-

272 associated proteins takes place (84). These highly dynamic plasma membrane SRDs in fungi often

273 contain ergosterol and sphingolipids. To determine whether loss of septins might affect one of the major

274 SRD-associated lipids, each septin mutant was challenged by drug treatments disrupting the ergosterol

275 biosynthesis pathway in spore dilution assays (Fig 5). These assays included an azole treatment

276 (Itraconazole), an allylamine treatment (Terbinafine), and a polyene treatment (Natamycin), which each

277 impact a different step in ergosterol biosynthesis (Fig 5A). Allylamines (Terbinafine) inhibit the

278 conversion of squalene to squalene epoxide, azoles (Itraconazole) inhibit the conversion of lanosterol to

279 4,4-dimethylcholesta-8,14,24-trienol, and polyenes bind directly to ergosterol (85).

280 The $\Delta a s p A^{c d c 11}, \Delta a s p B^{c d c 3}, \Delta a s p C^{c d c 12}$, and $\Delta a s p D^{c d c 10}$ mutants were more resistant to Itraconazole and

281 Terbinafine than WT or $\Delta a s p E$. Only $\Delta a s p A^{c d c 11}$ and $\Delta a s p E$ showed strong resistance to Natamycin

282 treatment (Fig 5B). The addition of exogenous 50 $\mathrm{MM}$ ergosterol was able to fully remediate the

283 sensitivity of all null mutant strains and WT to Itraconazole, Terbinafine, and Natamycin, suggesting

284 ergosterol was indeed the primary lipid component disrupted by these treatments (Fig 5C). These data

285 suggest that all five septins are involved in monitoring ergosterol metabolism and/or deposition. 
A

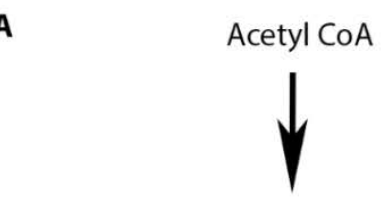

$\begin{aligned} & \text { Allylamines } \\ & \text { (Terbinafine) }\end{aligned}-1$

Squalene epoxide

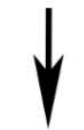

Lanosterol

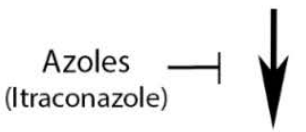

Polyenes
(Natamycin) - Ergosterol
B

C control

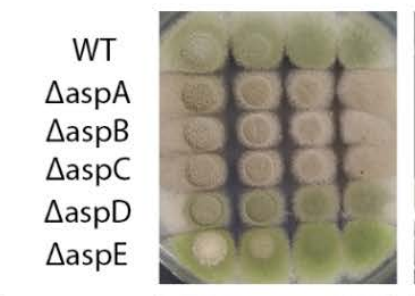

WT

$\triangle \mathrm{aspA}$

$\triangle \mathrm{aspB}$

$\triangle \mathrm{aspC}$

$\triangle \mathrm{aspD}$

$\triangle \mathrm{aspE}$

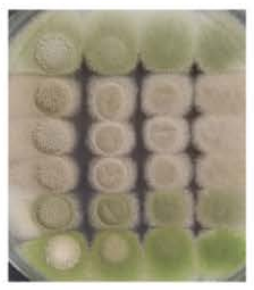

Ergosterol 50uM

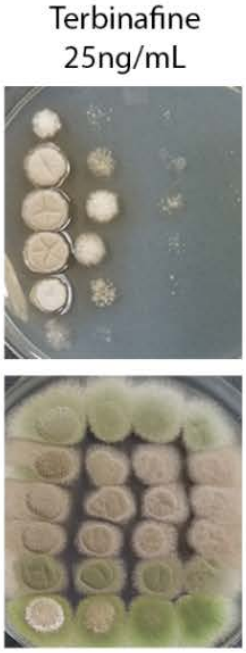

Terbinafine

$25 \mathrm{ng} / \mathrm{mL}$

$+$

Ergosterol 50uM
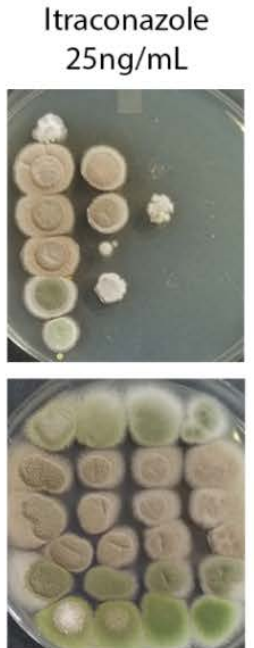

Itraconazole $25 \mathrm{ng} / \mathrm{mL}$

Ergosterol 50uM
Natamycin $1.7 \mathrm{ug} / \mathrm{mL}$
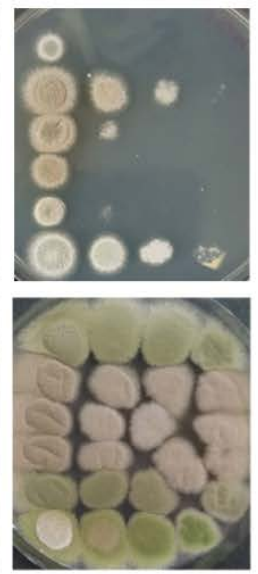

Natamycin $1.7 \mathrm{ug} / \mathrm{mL}$

Ergosterol 50uM

Fig 5. Core septin null mutants show increased resistance to disruption of ergosterol biosynthesis and $\triangle a s p A^{c d c 11}$ and $\triangle a s p E$ show increased resistance to the ergosterol-binding polyene drug

Natamycin. (A) A simplified schematic diagram of the ergosterol biosynthesis pathway, showing where allylamines, azoles, and polyene antifungal agents affect each step (85). (B) WT and septin null mutants $\triangle a s p A^{c d c 11}, \Delta a s p B^{c d c 3}, \Delta a s p C^{c d c 12}, \Delta a s p D^{c d c 10}$, and $\Delta a s p E$, were tested for sensitivity by spotting decreasing spore concentrations on solid media with or without the ergosterol biosynthesis-disturbing agents Terbinafine, Itraconazole, and Natamycin at concentrations shown. (C) Remediation of sensitivity to ergosterol biosynthesis-inhibiting treatments. WT and septin null mutants were tested for the ability of exogenous ergosterol $(50 \mu \mathrm{M})$ to remediate sensitivity to ergosterol biosynthesis-disturbing agents. Differences in colony color result from changes in spore production, spore pigment, and production of secondary metabolites under stress. Spore concentrations were $\left[10^{7}\right.$ conidia $/ \mathrm{mL}-10^{4}$ conidia $\left./ \mathrm{mL}\right]$ for all assays in figure. $\mathrm{N}=3$

\section{Core hexamer septin null mutants show altered sensitivity to disruption of sphingolipid biosynthesis.}

Sphingolipids are a class of plasma membrane lipids which has been shown to be associated with sterol-

rich domains, along with sterols and phosphoinositides, and therefore are possible targets for septin- 
step of the biosynthetic pathway, converting palmitoyl-coA and serine to 3-ketodihydrosphingosine,

preventing the accumulation of downstream intermediates, such as ceramides and sphingoid bases like phytosphingosine (PHS), as well as complex sphingolipids at the final steps of the pathway (Fig 6A) (37, 47). Aureobasidin A inhibits IPC synthase, disrupting the conversion of inositolphosphorylceramide from phytoceramide, and consequently causing the accumulation of intermediates such as phytosphingosine, which has been shown to be toxic at high concentrations (Fig 6A) (86).

314 The core hexamer null mutants $\Delta a s p A^{c d c 11}, \Delta a s p B^{c d c 3}$, and $\Delta a s p C^{c d c 12}$ were more sensitive to Myriocin than the other septin null mutants or WT (Fig 6B). $\Delta a s p A^{c d c 11}, \Delta a s p B^{c d c 3}$, and $\Delta a s p C^{c d c 12}$ were also more resistant to AbA than null mutants of the octamer-exclusive septin $\Delta a s p D^{c d c 10}$, the noncore septin $\Delta a s p E$,

317 or WT (Fig 6B). Strikingly, the addition of exogenous PHS $(15 \mu \mathrm{M})$ to the Myriocin treatment fully

318 remediated the sensitivity of $\Delta a s p A^{c d c 11}, \Delta a s p B^{c d c 3}$, and $\Delta a s p C^{c d c 12}$ (Fig 6C). $\Delta a s p A^{c d c 11}, \Delta a s p B^{c d c 3}$, and $319 \Delta a s p C^{c d c 12}$ were also more resistant to higher concentrations $(20 \mu \mathrm{M})$ of the phytosphingosine 320 intermediate (PHS), which has been shown to be toxic at high concentrations (Fig 6C).

321 To address whether cell wall and plasma membrane defects might be associated with one another in 322 septin null mutants, a combinatory drug treatment approach was taken. Sublethal concentrations of CFW $323(2 \mu \mathrm{g} / \mathrm{mL})$ and Myriocin $(10 \mu \mathrm{g} / \mathrm{mL})$, in which all strains grew at every spore concentration, were 324 combined (Fig 6D). When combined, the two drugs resulted in additive, colony-level growth defects for $325 \Delta a s p A^{c d c 11}, \Delta a s p B^{c d c 3}$, and $\Delta a s p C^{c d c 12}$. This sensitivity to both drugs in combination was remediated by 326 the addition of exogenous PHS. Surprisingly the addition of exogenous sorbitol, which had fully 327 remediated the hypersensitivity of the septin null mutants to all previously tested cell wall-disturbing 328 agents, resulted in a more dramatic growth defect in media containing only Myriocin, or in combination 329 with CFW (Fig 6C and D). These data together suggest that the core hexamer septins AspA ${ }^{\text {Cdc11, }}$ $330 \mathrm{AspB}^{\mathrm{Cdc3}}$, and $\mathrm{AspC} \mathrm{Cdc12}^{\mathrm{C}}$ may monitor sphingolipid metabolism. They further suggest that the hexamer 331 septins may signal sphingolipid status to the cell wall integrity pathway, and that this signal is required 332 for proper cell wall integrity function. 
A

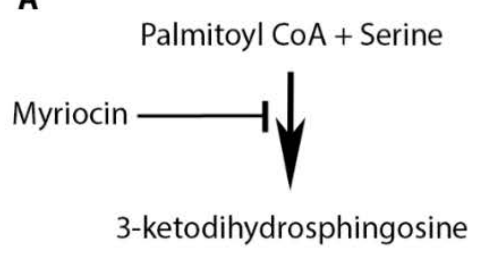

$\downarrow$

Dihydrosphingosine

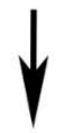

Phytosphingosine<smiles>CC(C)CC(C)(C)C</smiles>

Phytoceramide

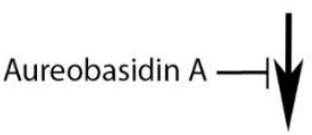

Complex Sphingolipids
B

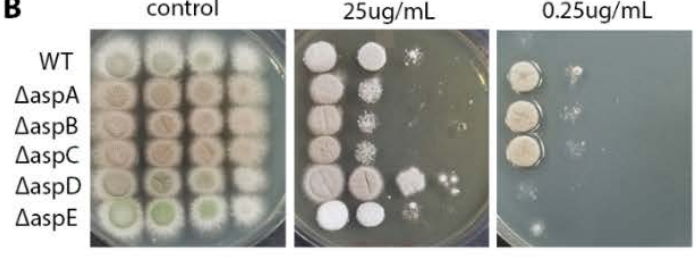

Fig 6. Septin core hexamer null mutants show altered sensitivity to agents which disrupt sphingolipid biosynthesis. (A) A simplified diagram of the sphingolipid biosynthesis pathway in $A$. nidulans (87). (B) Solid media spotting assay. WT and septin null mutants, $\Delta a s p A^{\text {cdc11 }}, \Delta a s p B^{c d c 3}, \Delta a s p C$ ${ }^{c d c 12}, \Delta a s p D^{c d c 10}$, and $\Delta a s p E$ were tested for sensitivity by spotting decreasing spore concentrations on solid media with or without sphingolipid biosynthesis-disturbing agents Myriocin and Aureobasidin A. (C) Remediation of sensitivity to sphingolipid biosynthesis-disturbing agents. WT and septin null mutants were tested for remediation of sensitivity to sphingolipid biosynthesis-disturbing agents by spotting decreasing spore concentrations on solid media amended with exogenous phytosphingosine (PHS) intermediate $(15 \mu \mathrm{M})$ or $1.2 \mathrm{M}$ sorbitol, with or without Myriocin. (D) Combinatory treatment with cell wall and sphingolipid biosynthesis-disturbing agents. WT and septin null mutants were tested for sensitivity to cell wall and sphingolipid biosynthesis-disturbing agents in combination, by spotting decreasing spore concentrations on solid media with or without 'sub-lethal' concentrations of Calcofluor White (CFW), Myriocin, or CFW + Myriocin, amended with either exogenous phytosphingosine intermediate $(15 \mathrm{uM})$ or $1.2 \mathrm{M}$ sorbitol. Differences in colony color result from changes in spore production, spore pigment, and production of secondary metabolites under stress. Spore concentrations were $\left[10^{7}\right.$ conidia/mL $-10^{4}$ conidia/mL $]$ for all assays in figure. $\mathrm{N}=3$

Localization of core hexamer septins $A s p A^{C d c 11}$ and $A s p B^{C d c 3}$ is disrupted by sphingolipid inhibitors.

We predicted that since core septin hexamer null mutants $\Delta a s p A^{c d c 11}, \Delta a s p B^{c d c 3}$, and $\Delta a s p C^{c d c 12}$ were sensitive to drugs which inhibit sphingolipid biosynthesis the localization of these septins might be 
355 altered when exposed to the same treatments. We examined whether disruption of sphingolipid

356 biosynthesis causes changes in septin localization using live-cell imaging with fluorescence microscopy.

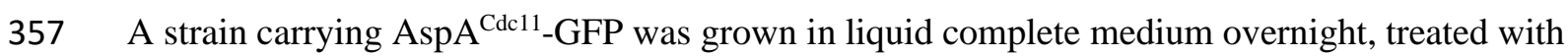

358 exogenous PHS (15 $\mu \mathrm{M})$, and imaged for 3 hours post-treatment. There was a dynamic shift in septin

359 localization under PHS treatment over the course of the experiment, compared to the vehicle control. The

360 septin-GFP signal shifted from a relatively homogenous cortical localization along the hyphal tips to a

361 more stochastic, punctate localization along the entire length of hyphae (Fig 7, middle panel).

362 In contrast to these results, treatments with the ergosterol-binding polyene, Filipin III $(25 \mu \mathrm{g} / \mathrm{mL})$ and

363 phosphatidylinositol 3-kinase inhibitor, Wortmannin $(20 \mu \mathrm{g} / \mathrm{mL})$, did not affect the localization pattern of

364 septins as dramatically as the PHS treatment (Fig 7, left- and right-most panels). Similar patterns of

365 aberrant localization were observed under PHS treatments for AspB ${ }^{\text {Cdc3 }}$-GFP (S5 Fig). Myriocin

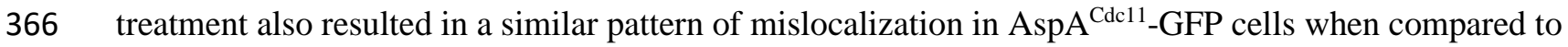

367 treatment with PHS (S6 Fig). These results suggest sphingolipid content and/or distribution within the

368 plasma membrane contributes to the localization and stability of core hexamer septins at the plasma

369 membrane and that ergosterol and phosphoinositides may not be as vital for this process. 


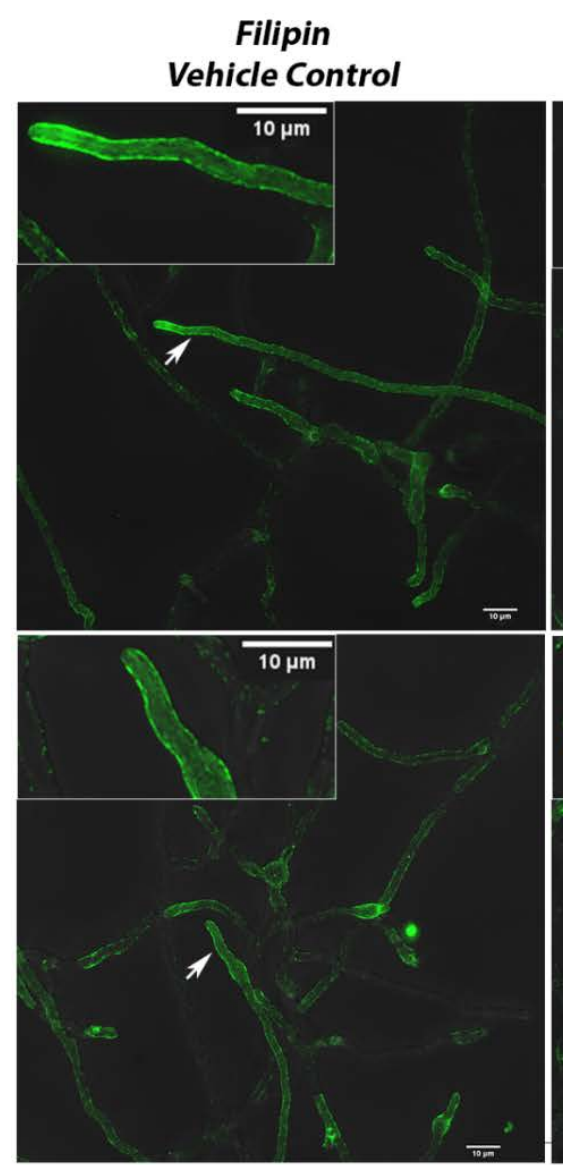

Filipin

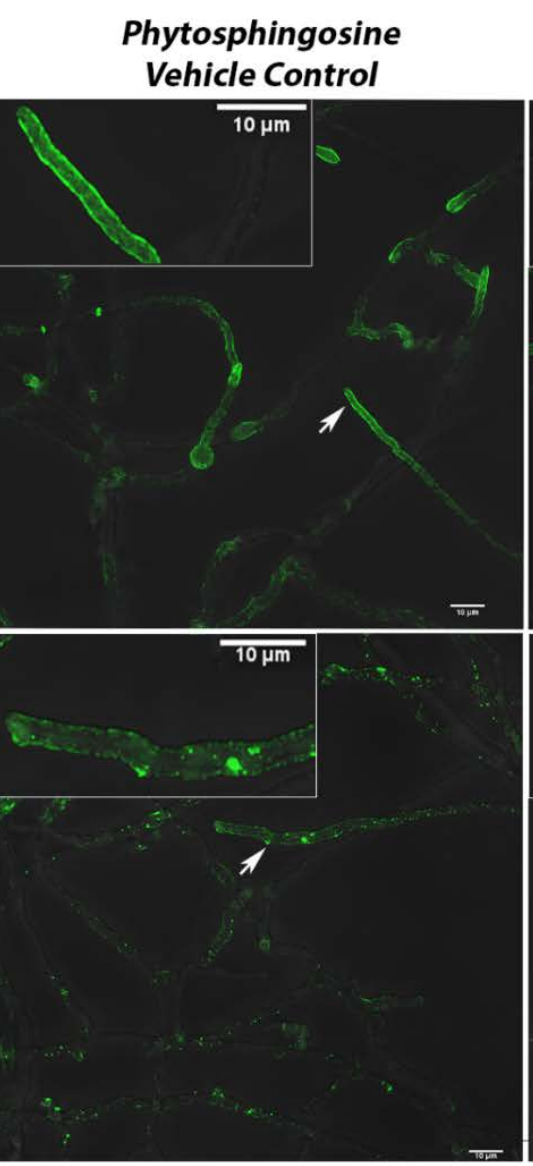

Phytosphingosine

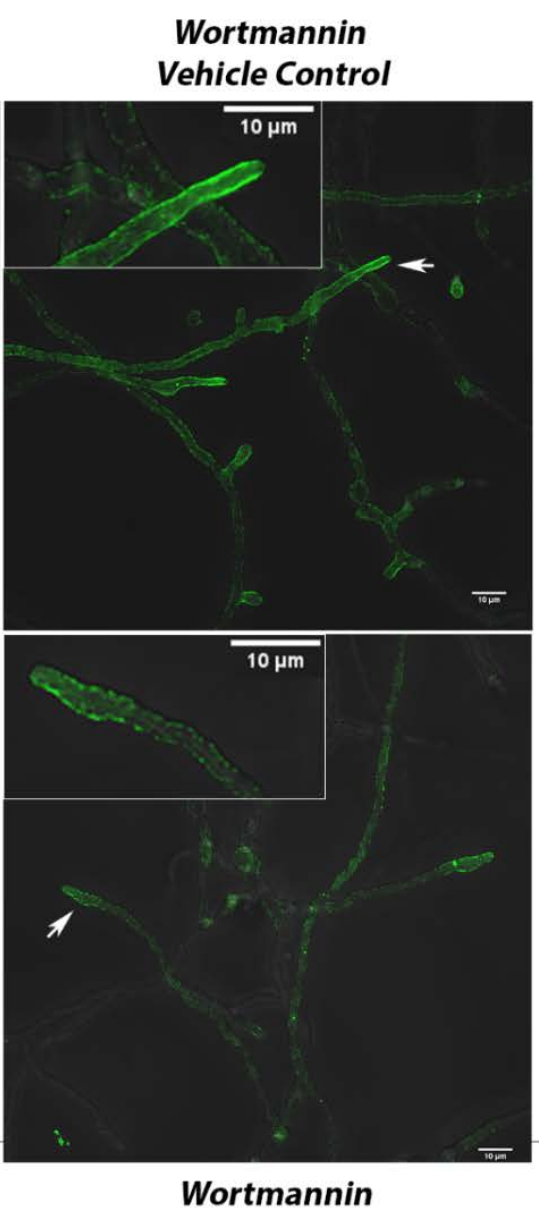

Wortmannin Vehicle Contro

371

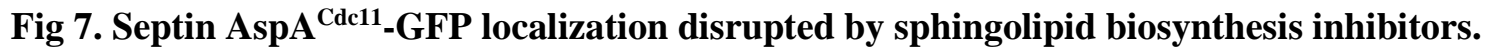

374

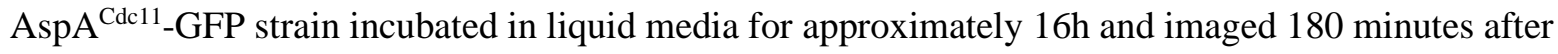
replacing with fresh media containing sterol, sphingolipid, and phosphoinositide-disturbing agents,

375 Filipin, phytosphingosine, and Wortmannin, respectively. Representative images are shown from three independent biological replicates with $\geq 100$ cells observed. (Top Row) Vehicle controls for each treatment. (Bottom Row) Filipin $(25 \mu \mathrm{g} / \mathrm{mL})$, phytosphingosine $(15 \mu \mathrm{M})$, and Wortmannin $(20 \mu \mathrm{g} / \mathrm{mL})$ treatments respectively. Insets show enlarged section of micrographs from each picture to better visualize pattern of fluorescence signal. Imaging conducted with Deltavision I deconvolution inverted fluorescence microscope. White arrows denote hyphae which are highlighted in enlarged images. Scale bars $=10 \mu \mathrm{m}$. $\mathrm{N}=3$

\section{Discussion}

Our data show that $A$. nidulans septins play roles in both plasma membrane and cell wall integrity and that distinct subgroups of septins carry out these roles. Previous work has shown that the five septins of $A$. 
nidulans septins form hexamers (AspA ${ }^{\text {Cdc11 }}, \mathrm{AspB}^{\mathrm{Cdc3}}$, and AspC $\left.\mathrm{Cdc12}^{\mathrm{Cd}}\right)$ and octamers $\left(\right.$ Asp $\mathrm{A}^{\mathrm{Cdc11}}$, $\mathrm{AspB}^{\mathrm{Cdc3}}$, AspC $\mathrm{Cdc12}^{\mathrm{C}}$, and $\mathrm{AspD}^{\mathrm{Cdc10}}$ ) and that the noncore septin AspE does not appear to be a stable member of a heteropolymer (20). The current work suggests that though all septins are involved in coordinating cell wall and membrane integrity, the roles of hexamers, octamers, and the noncore septin are somewhat different. Core hexamer septins appear to be most important for sphingolipid metabolism, all five septins appear to be involved in ergosterol metabolism, and core septins are most important for cell wall integrity pathway with the noncore septin possibly playing a minor role. As summarized in

393 Figure 8 and discussed in more detail below, our previous and current data are consistent with a model in 394 which: (A) All five septins assemble at sites of membrane and cell wall remodeling in a sphingolipid395 dependent process; (B) All five septins recruit and/or scaffold ergosterol and the core hexamer septins recruit and/or scaffold sphingolipids and associated sensors at these sites, triggering changes in lipid metabolism; and (C) The core septins recruit and/or scaffold cell wall integrity machinery to the proper locations and trigger changes in cell wall synthesis. The noncore septin might play a minor role in this process.

Septins assemble at sites of membrane and cell wall remodeling in a sphingolipid-dependent process.

401 We hypothesize that sterol rich domain-associated lipids (ergosterol, sphingolipids, and

402 phosphoinositides) recruit or facilitate binding and assembly of septins on the membrane, with

403 sphingolipids contributing more to the stabilization of septins than other SRD-associated lipids in A.

nidulans (Fig 8A). Previous studies showed preferential in vitro binding of yeast core septin orthologues to the phosphoinositides PIP2, PI(4,5)P2, PI(5)P, and PI(4)P, however our treatments with Wortmannin and Filipin III, known disruptors of phosphoinositides and ergosterol respectively (88-92), did not affect

407 septin localization as dramatically as sphingolipid-disturbing treatments (Fig 7,S3, and S4 Fig). The 
411 microdomains showed that relatively minor differences in sphingolipid structure can have significant

412 effects on sterol and phospholipid interactions, consequently resulting in major changes in membrane

413 properties $(93,94)$. Perhaps sphingolipids (and ergosterol to a lesser extent) help to stabilize SRDs in a

414 way that facilitates assembly of septin filaments and higher order structures via diffusion, collision, and

415 annealing as proposed by Bridges et al (2014) (41-43, 95). Consistent with this idea, A. nidulans AspB

416 filaments have been shown to move along the plasma membrane, break apart, and 'snap' together in a

417 way that suggests collision and annealing (21).

Septins recruit and/or scaffold lipids and associated sensors, triggering changes in lipid metabolism.

419 Consistent with a role for septins in modulating membrane composition, all septin null mutants were

420 resistant to ergosterol-disrupting treatments and core hexamer septin mutants were affected by disruption

421 of sphingolipid biosynthesis (Fig 5A-B, Fig 6). Based on proposed mechanisms for septins as diffusion

422 barriers or organizational scaffolds of membrane-associated proteins in yeast and smut fungi, we propose

423 septins monitor lipid microdomain composition and/or organization in filamentous ascomycetes (49,

424 51)(Fig 8B). Septins do not have a transmembrane domain, a feature that often defines established

425 membrane 'sensors' that monitor local lipid environments (96, 97); however, septins share a highly

426 conserved polybasic domain proposed to facilitate septin-membrane interactions $(44,98)$. In addition to

427 the polybasic domain, septins have recently been shown to contain an amphipathic helix motif which has

428 been implicated in septin sensing of membrane curvatures (99). Given that septins have been shown to

429 assemble into non-polar higher order structures along the plasma membrane (95), septin assembly itself

430 might be the mechanism by which lipid composition and protein organization is monitored at the

431 cytosolic face of membranes. Perhaps septin assemblies that pass specific size or geometric thresholds

432 trigger signaling through MAPK and other pathways. 
bioRxiv preprint doi: https://doi.org/10.1101/2020.02.09.940718; this version posted December 9, 2020. The copyright holder for this preprint (which was not certified by peer review) is the author/funder, who has granted bioRxiv a license to display the preprint in perpetuity. It is made available under aCC-BY-NC 4.0 International license.

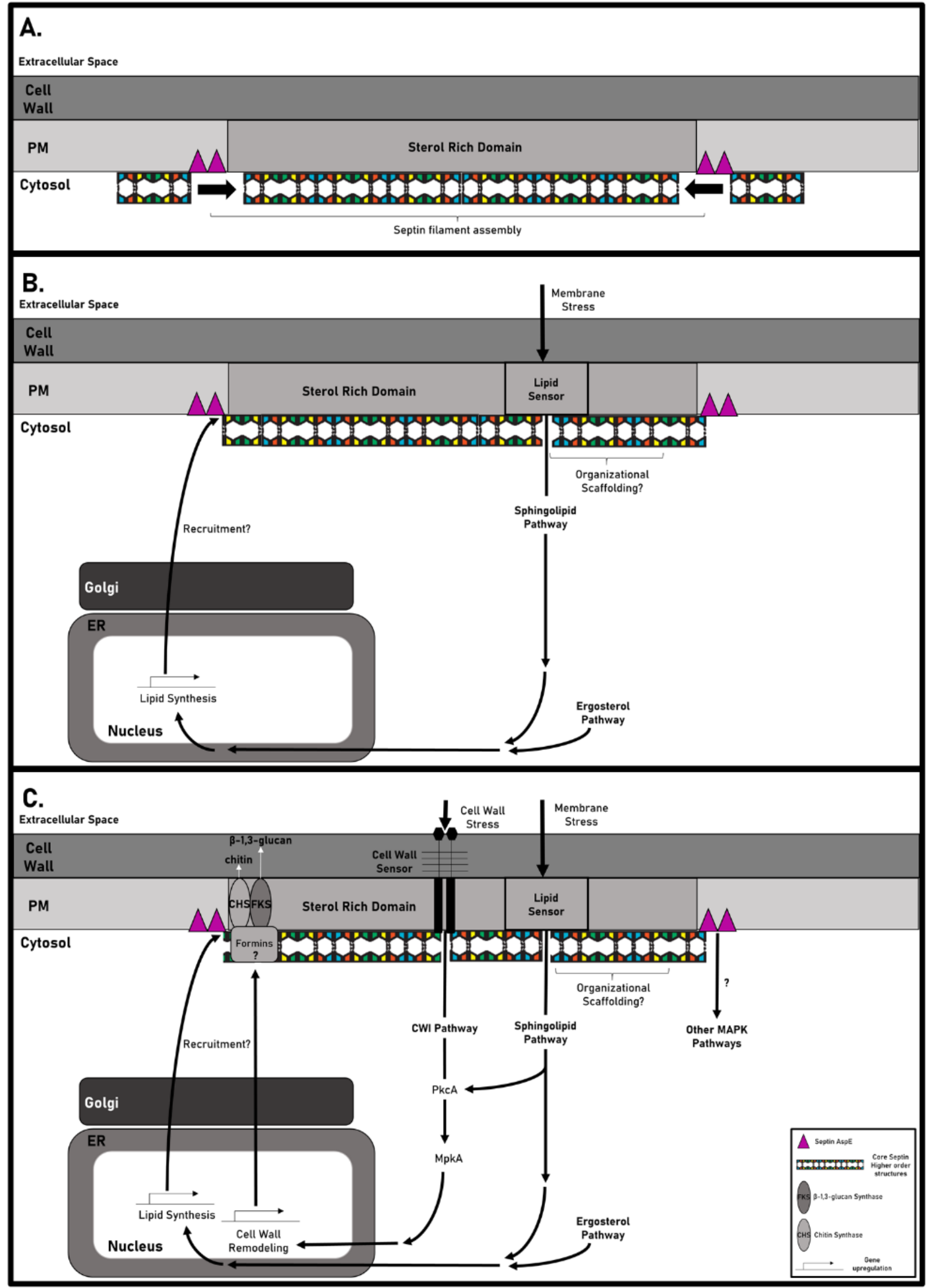


Figure 8. Model for septin modulation of cell wall and plasma membrane integrity through septins are involved in cell wall and membrane integrity coordination. The core septins that participate in hexamers appear to be most important for sphingolipid metabolism while all septins appear to be involved in ergosterol metabolism and cell wall integrity. Because SRDs contain both sphingolipids and ergosterol and because it is not yet clear how subgroups of septins interact with each other at SRDs, we show all core septins in our model without distinguishing hexamers and octamers. In this model, septins are proposed to colocalize with SRDs in a manner which promotes: (A) assembly of septins into higher order structures along the membrane at sites of polarized growth or cell wall/PM remodeling; (B) the recruitment and/or scaffolding of lipids and associated membrane-bound sensors to monitor membrane composition and/or stress. The status of membrane composition is relayed to the nucleus where it triggers changes in expression of genes responsible for lipid metabolism; and (C) the recruitment and/or scaffolding of cell wall integrity pathway machinery to monitor cell wall composition and stress, followed by the recruitment and/or scaffolding of cell wall synthases, possibly with the help of other septininteracting proteins such as formins. Question marks (?) denote speculative processes or interactions

changes in cell wall synthesis. In addition to monitoring and relaying information about the membrane, septins clearly have a role in normal cell wall growth and remediation of cell wall stress via the cell wall integrity pathway. We propose a major role of the core septins is to recruit and/or organize integral proteins to sites of polarized growth or remodeling at the cell cortex to ensure cell wall integrity pathway functions are carried out (Fig 8C). The sensitivity to cell wall-disturbing agents, altered cell wall composition, and altered polysaccharide deposition in the core septin null mutants (Fig 1-2 and S1-S2 
467 integrity pathway sensors or kinases could exist. This interpretation is consistent with studies in yeast

468 showing Bni4 (ANID_00979), a formin which is phosphorylated by and functions downstream of MAPK

469 Slt2 ${ }^{m p k A}$, directly interacts with the core septin orthologues in order to recruit chitin synthases to the bud

470 neck (111-113). Though the involvement of AspE in the cell wall integrity pathway appears to be minor

471 based on the very mild sensitivity of the null mutant to Congo Red (Fig 1), sensitivity to TOR and cAMP-

472 PKA pathway inhibitors suggests that AspE might participate indirectly through MAPK pathway cross-

473 talk (Fig S4). It could be that in the septin null mutants, septin filaments are not able to properly

474 assemble and monitor lipid composition and protein organization at the cytosolic face of membranes,

475 resulting in defects in plasma membrane and cell wall integrity (Fig 8).

476 Though we have discussed membrane and cell wall integrity separately, it is possible that that membrane

477 defects in the septin null mutants contribute to the observed cell wall changes or that cell wall defects

478 contribute to the observed changes in lipid metabolism. When septin deletion mutants were challenged

479 with both membrane-disturbing and cell wall-disturbing agents in combination, remediation of the lipid

480 defect (via PHS) restored proper growth, but remediation of the cell wall defect (via sorbitol) did not

481 remediate lethality (Fig 6C-D). This suggests that there is a synergistic effect of disrupting sphingolipid

482 metabolism and cell wall architecture in septin null mutants and that septin-sphingolipid interactions are

483 required for roles in maintaining cell wall integrity.

484 Because $A$. nidulans septin deletion mutants are viable, we were able to systematically analyze the roles

485 of all septins in this organism. Our data show that all septins are required for proper coordination of lipid

486 metabolism, with core hexamer septins most important for sphingolipid metabolism and all septins

487 involved in ergosterol metabolism. Our data also show that the core septins are most important for cell

488 wall integrity pathway, but that these roles require sphingolipids. Based on our data we propose that

489 septins are critical for tight coordination of plasma membrane metabolism and cell wall synthesis during

490 normal development and response to exogenous stress and that the site of this coordination is likely 
SRDs. Future work will address how subgroups of septins interact with each other and with sphingolipids

492 and ergosterol in SRDs to coordinate the cell wall integrity response.

\section{Methods}

Spore Dilution Drug Sensitivity Assays

Strains used in this study are listed in S1 Table. Media used were previously described (114). conidia/mL, and then serial diluted 4-fold into separate Eppendorf tubes. All strains were inoculated in 10uL droplets in a grid pattern on petri plates containing $25 \mathrm{~mL}$ of solid (1.8\% agar) complete medium (CM) (1\% glucose, $0.2 \%$ peptone, $0.1 \%$ Casamino Acids, $0.1 \%$ yeast extract, trace elements, nitrate salts,

501 and $0.01 \%$ vitamins, $\mathrm{pH}$ 6.5; with amino acid supplements as noted) or minimal medium (MM)(1\%

502 glucose, trace elements, $1 \%$ thiamine, $0.05 \%$ biotin, $\mathrm{pH}$ 6.5; with amino acid supplements as noted) with

503 or without amended supplements. All incubations were conducted at $30^{\circ} \mathrm{C}$ as indicated for $3-4$ days

504 before images were taken. Sorbitol, $\mathrm{NaCl}$, and $\mathrm{KCl}$ were added at $2 \mathrm{M}, 1 \mathrm{M}$, and $1.5 \mathrm{M}$ respectively to

505 media before autoclaving. Stock solutions were prepared as follows: Blankophor BBH/Calcofluor White

506 (Bayer Corporation; standard-SV-2460; 25mg/mL in ddH20 and adjusted pH with 1M KOH until

507 solubilized), Congo Red (Fisher Scientific; Lot No.8232-6; 10mg/mL in ddH20), Caspofungin acetate

508 (1mg/mL in ddH20), Fludioxonil/Pestanal (2mg/mL in DMSO), and caffeine monohydrate (63.66mg/mL

509 in ddH20 and gently heated with stirring until solubilized); calcium chloride (2M in ddH20), EGTA

510 (0.5M in ddH20), Rapamycin/Sirolimus (1mg/mL in acetonitrile), FK-506 (5mg/mL in DMSO),

511 Natamycin (1.5mg/mL in MeOH), Itraconazole (10mg/mL in DMSO), Terbinafine (10mg/mL in DMSO).

512 Stock solutions of Myriocin $(5 \mathrm{mg} / \mathrm{mL})$, phytosphingosine $(1 \mathrm{mg} / \mathrm{mL})$, and Aureobasidin A $(1 \mathrm{mg} / \mathrm{mL})$

513 were prepared in DMSO, EtOH, and $\mathrm{MeOH}$ respectively and stored at -20C in the dark. Images of plates 
514 were captured using a cellular device with an 8.0 Megapixel camera, and subsequently processed using

515 Photoshop CS5 Version 12.0 X32.

517 Parental strains were coinoculated in CM liquid at 1 X10 $10^{5}$ conidia/mL supplemented with all parental

518 auxotrophic markers and allowed to incubate at $30^{\circ} \mathrm{C}$ for up to one week or until a thick mycelial mat had

519 formed. Mycelial mats were transferred to solid MM plates containing only shared auxotrophic markers

520 from the genetic backgrounds of each parental strain. Plates containing mycelial mats were parafilmed

521 and incubated in the dark at room temperature for up to 2 weeks or until mature cleistothecia form on the

522 mycelial mats. Multiple cleistothecia from each genetic cross were collected in water, diluted, and plated

523 onto solid media containing all auxotrophic supplements from each parental strain to allow growth of all

524 resulting progeny. Approximately 50 progenies were collected from each dissected cleistothecium, and

525 each colony was transferred to master plates, and replica-plated onto minimal media without any

526 supplements, to isolate prototrophic progeny. Five to ten progenies from each cross were then 3-phased

527 streaked to obtain single colony isolates for PCR verification. All progenies of genetic crosses were

528 verified by diagnostic PCR using KOD XTREME Hot Start DNA Polymerase (71975-3, EMD Millipore)

529 or OneTaq ${ }^{\circledR}$ Hot Start Quick-Load ${ }^{\circledR} 2$ X Master Mix with Standard Buffer (M0488L, New England

530 BioLabs inc.) according to manufacturer's instructions. Double mutant strains of $\Delta m p k A^{\text {slt2 }}$ were verified

531 for deletion of $m p k A^{\text {slt2 }}$ by amplification of entire gene using primers, MpkA-806-F' and MpkA-3779-R',

532 followed by SacI HF restriction enzyme digestion of PCR product to better visualize band sizes. All

533 progeny from chitin synthase and septin null mutant crosses were determined to be virtually identical to

534 each septin mutant parental strain in growth/morphology on a colony level and by microscopy, PCR-

535 verified for the deletion of each septin gene, and screened visually by fluorescence microscopy for the

536 presence of chitin synthase-GFP signal. Chitin synthase-GFP strains in a $\triangle \operatorname{asp} A^{\text {cdc11 }}$ genetic background

537 were verified for deletion of $a s p A^{c d c 11}$ by amplification of the entire gene using primers, AspA-KO-F' and 
AspA-KO-R', followed by XhoI restriction enzyme digestion for verification of band sizes. Strains and primer sets used in this study can be found in S1 and S2 Tables respectively.

541 Growth and preparation of cells were as previously reported (115). Conidia were inoculated on sterile 542 coverslips in $10 \mathrm{~mL}$ liquid complete or minimal media at $1 X 10^{5}$ conidia/mL and incubated at 30C in a small 543 petri dish for the specified amount of time. Cell walls were stained for chitin with Blankophor BBH (CFW) 544 (American Cyanamid, Wayne, NJ; 25mg/mL stock solution in ddH2O and pH adjusted by $1 \mathrm{M} \mathrm{KOH}$ until solubilized; working solution made by diluting stock solution by $100 \mathrm{X}$ and $8 \mathrm{ul}$ dissolved in $5 \mathrm{~mL}$ ddH20 prepared fresh for working solution and used immediately), $\beta$-(1,3)-glucans were stained with aniline blue

547 (stock solution prepared fresh to $10 \mathrm{mg} / \mathrm{mL}$ final concentration in ddH20; working solution prepared at $135.55 \mu \mathrm{M}$ in $50 \mathrm{mM}$ phosphate buffer, $\mathrm{pH}$ adjusted to 9.5 with $5 \mathrm{M} \mathrm{KOH}$, and used immediately; coverslips stained for 5 minutes in the dark at RT). Live cell imaging experiments tracking septin localization were conducted using Filipin III (stock solution prepared at 5mg/mL in DMSO; working solution was used at $55125 \mu \mathrm{g} / \mathrm{mL}$ final concentration in liquid complete media), phytosphingosine (working solution was used at $55215 \mu \mathrm{M}$ in liquid complete media), and Wortmannin (stock solution prepared to $2 \mathrm{mg} / \mathrm{mL}$ in DMSO; working 553 solution was used at $20 \mathrm{ug} / \mathrm{mL}$ in liquid complete media). Vehicle controls and conducted at $\leq 1 \% \mathrm{w} / \mathrm{v}$ in 554 liquid media. Imaging was performed in the Biomedical Microscopy Core at the University of Georgia.

555 Microscopy was carried out using Zeiss Axioplan microscope and Zeiss Axiocam MRc charge-coupled 556 device camera and software, as well as Deltavision I Deconvolution Inverted Microscope and LSM880 557 Confocal Fluorescent microscope with Diode laser (405nm), Argon (458, 488, 514nm) and HeNe (543, 558 633nm) laser lines. All micrograph comparisons between treatments imaged with identical microscope 559 settings. Subsequent image analysis and scale bars added to micrographs, using ImageJ software 1.48v, 560 Java 1.6.0_20 (64-bit) or Zen 2.3 imaging software, and final figures compiled in Photoshop CS5 561 software version 12.0 x32. 
563 Max projection images and scale bars were made in each micrograph in Zen (Black) Edition.

564 Micrographs were false colored black and white, line scans were drawn by hand through hyphal tips, line

565 scan profiles were generated in Zen 3.1 (Blue) imaging software, and associated date points for each

566 profile were exported to Microsoft Excel version 2004 (Build 12730.20270) to create graphs and conduct

567 statistical analysis. Figures were generated using Adobe Photoshop 2020 Version: 21.1.2.

Quantification of chitin synthase-GFP and CFW fluorescence signal by line scans

569 Max projection images were made for each micrograph, line scans were drawn by hand through hyphal

570 branch tips in ImageJ software 1.48v, and data points were exported to Microsoft Excel version 2004

571 (Build 12730.20270) to create graphs and conduct statistical analysis. Figures were assembled using

572 Adobe Photoshop 2020 Version: 21.1.2.

574 Cell walls were isolated from a protocol based on (Bull, 1970); cell wall extraction and lyophilization 575 were conducted as previously described in (Guest and Momany, 2000) with slight modifications listed in 576 full procedure here. A single batch of complete media (recipe described above) was autoclaved and 577 supplements added: Arginine, Methionine, Pyridoxine, and Riboflavin, were added to single flask and 578 then distributed to individual flasks to be inoculated. 1 X $10^{4}$ conidia/mL were inoculated in 2 flasks each 579 of 100mL liquid Complete Media. Flasks were incubated at 30C in orbital shaker at 200rpm for 48 hours. 580 Mycelia was gravity filtrated, then vacuum-filtrated through \#42 Whatman filter paper, subsequently 581 washed with 50mL each of chilled ddH20 to remove residual media and stored at -80C until completely 582 frozen. Mycelium from each sample was allowed to thaw on ice and then washed sequentially with $50 \mathrm{~mL}$ 583 chilled ddH20 and 0.5M NaCl. Fungal hyphal mats were transferred to mortar and pestle, then 584 subsequently flash frozen in liquid nitrogen and ground in chilled Tris/EDTA Disruption Buffer (DB; 585 20mM Tris, 50mM EDTA, pH 8.0) with pre-chilled mortar and pestle. Samples were monitored by 
microscopy under 60X or 100X objective until hyphal ghosts were evident. Cell walls were separated by

587 centrifugation at $13,800 \mathrm{~g}$ for $10 \mathrm{~min}$ at 4 C. Cell pellet was placed in a beaker with $40-100 \mathrm{~mL}$ of chilled

588 Tris/EDTA buffer and stirred at 4C for 12 hours. Cell pellet was collected by centrifugation as above and

589 stirred again with $100 \mathrm{~mL}$ chilled ddH20 at 4C for 4 hours. Cell wall materials was collected by vacuum

590 filtration, frozen at $-80 \mathrm{C}$, lyophilized to dryness, and stored at room temperature $\left(25^{\circ} \mathrm{C}\right)$ for further

591 analysis.

To determine the glycosyl linkages, the samples were acetylated using pyridine and acetic anhydride in order to get better solubility, before two rounds of permethylation using sodium hydroxide (15 min) and methyl iodide (45 min). The permethylated material was hydrolyzed using 2M TFA (2 h in sealed tube at $121^{\circ} \mathrm{C}$ ), reduced with $\mathrm{NaBD}_{4}$, and acetylated using acetic anhydride/pyridine. The resulting PMAAs (Neutral sugars) were analyzed on an Agilent 7890A GC interfaced to a 5975C MSD (mass selective detector, electron impact ionization mode); separation was performed on a $30 \mathrm{~m}$ Supelco SP-2331 bonded phase fused silica capillary column using Supelco SP-2331 fused silica capillary column (30 m x 0,25 mm ID). The PMAAs of amino sugars were separated on Supelco Equity-1 column (30 m x 0.25 mm ID).

601 Further, the relative quantities of respective glycosyl linkages were calculated by integrating the peak area

602 of respective peak. Since the neutral and amino sugars were analyzed on different instruments, the peak 603 area of amino sugars was normalized with 4-Glc peak, which is prominent in both the instruments and the 604 integrated and normalized peak areas were pooled together to calculate the relative percentage of individual 605 linkages. Two independent, biological replicates were conducted for this analysis and processed in tandem. 606 The average area (\%) of detected linkages of one representative data set is included in the graphs to show 607 relative differences between cell wall polysaccharides between samples. 


\section{Acknowledgements}

611 These studies were supported by the University of Georgia Franklin College of Arts and Sciences support

612 to MM and Plant Biology Department support to AM. Chitin synthase strains were generously provided

613 by Dr. Hiroyuki Horiuchi in the Department of Biotechnology at the University of Tokyo. The glycosyl

614 linkage analysis was supported by a Chemical Sciences, Geosciences and Biosciences Division, Office of

615 Basic Energy Sciences, U.S. Department of Energy grant (DE-SC0015662) to Parastoo Azadi at the

616 Complex Carbohydrate Research Center at UGA. 


\section{References}

619 1. Shaw JA, Mol PC, Bowers B, Silverman SJ, Valdivieso MH, Duran A, et al. The function of

620 chitin synthases 2 and 3 in the Saccharomyces cerevisiae cell cycle. J Cell Biol. 1991;114(1):111-23.

$6212 . \quad$ Bulawa CE, Miller DW, Henry LK, Becker JM. Attenuated virulence of chitin-deficient mutants

622 of Candida albicans. Proc Natl Acad Sci U S A. 1995;92(23):10570-4.

623 3. Sudoh M, Yamazaki T, Masubuchi K, Taniguchi M, Shimma N, Arisawa M, et al. Identification

624 of a novel inhibitor specific to the fungal chitin synthase. Inhibition of chitin synthase 1 arrests the cell

625 growth, but inhibition of chitin synthase 1 and 2 is lethal in the pathogenic fungus Candida albicans. J

626 Biol Chem. 2000;275(42):32901-5.

627 4. Munro CA, Gow NA. Chitin synthesis in human pathogenic fungi. Med Mycol. 2001;39 Suppl

$628 \quad 1: 41-53$.

629 5. Schmidt M. Survival and cytokinesis of Saccharomyces cerevisiae in the absence of chitin.

630 Microbiology. 2004;150(Pt 10):3253-60.

631 6. Orlean P. Architecture and biosynthesis of the Saccharomyces cerevisiae cell wall. Genetics.

632 2012;192(3):775-818.

633 7. Gladfelter AS, Pringle JR, Lew DJ. The septin cortex at the yeast mother-bud neck. Curr Opin

634 Microbiol. 2001;4(6):681-9.

635 8. Longtine MS, Bi E. Regulation of septin organization and function in yeast. Trends Cell Biol.

636 2003;13(8):403-9.

637 9. Caudron F, Barral Y. Septins and the lateral compartmentalization of eukaryotic membranes. Dev 638 Cell. 2009;16(4):493-506. 
10. Takizawa PA, DeRisi JL, Wilhelm JE, Vale RD. Plasma membrane compartmentalization in

640 yeast by messenger RNA transport and a septin diffusion barrier. Science. 2000;290(5490):341-4.

641 11. Dobbelaere J, Barral Y. Spatial coordination of cytokinetic events by compartmentalization of the

642 cell cortex. Science. 2004;305(5682):393-6.

643 12. Oh Y, Bi E. Septin structure and function in yeast and beyond. Trends Cell Biol. 2011;21(3):141-

6448.

645 13. Glomb O, Gronemeyer T. Septin Organization and Functions in Budding Yeast. Front Cell Dev

646 Biol. 2016;4:123.

647 14. Trimble WS, Grinstein S. Barriers to the free diffusion of proteins and lipids in the plasma 648 membrane. J Cell Biol. 2015;208(3):259-71.

649 15. McMurray MA, Bertin A, Garcia G, 3rd, Lam L, Nogales E, Thorner J. Septin filament formation 650 is essential in budding yeast. Dev Cell. 2011;20(4):540-9.

651 16. Sirajuddin M, Farkasovsky M, Hauer F, Kuhlmann D, Macara IG, Weyand M, et al. Structural 652 insight into filament formation by mammalian septins. Nature. 2007;449(7160):311-5.

653 17. Bertin A, McMurray MA, Grob P, Park SS, Garcia G, 3rd, Patanwala I, et al. Saccharomyces

654 cerevisiae septins: supramolecular organization of heterooligomers and the mechanism of filament

655 assembly. Proc Natl Acad Sci U S A. 2008;105(24):8274-9.

656 18. McMurray MA, Thorner J. Turning it inside out: the organization of human septin hetero-

657 oligomers. Cytoskeleton (Hoboken). 2019.

658 19. Pan F, Malmberg RL, Momany M. Analysis of septins across kingdoms reveals orthology and 659 new motifs. BMC Evol Biol. 2007;7:103. 
660 20. Hernandez-Rodriguez Y, Masuo S, Johnson D, Orlando R, Smith A, Couto-Rodriguez M, et al.

661 Distinct septin heteropolymers co-exist during multicellular development in the filamentous fungus

662 Aspergillus nidulans. PLoS One. 2014;9(3):e92819.

663 21. Hernandez-Rodriguez Y, Hastings S, Momany M. The septin AspB in Aspergillus nidulans forms

664 bars and filaments and plays roles in growth emergence and conidiation. Eukaryot Cell. 2012;11(3):311-

66523.

666 22. Fukuda K, Yamada K, Deoka K, Yamashita S, Ohta A, Horiuchi H. Class III chitin synthase

667 ChsB of Aspergillus nidulans localizes at the sites of polarized cell wall synthesis and is required for

668 conidial development. Eukaryot Cell. 2009;8(7):945-56.

669 23. Blankenship JR, Fanning S, Hamaker JJ, Mitchell AP. An extensive circuitry for cell wall

670 regulation in Candida albicans. PLoS Pathog. 2010;6(2):e1000752.

671 24. Mostowy S, Cossart P. Septins: the fourth component of the cytoskeleton. Nat Rev Mol Cell Biol.

$672 \quad 2012 ; 13(3): 183-94$.

673 25. Wiederhold NP, Kontoyiannis DP, Prince RA, Lewis RE. Attenuation of the activity of

674 caspofungin at high concentrations against candida albicans: possible role of cell wall integrity and

675 calcineurin pathways. Antimicrob Agents Chemother. 2005;49(12):5146-8.

676 26. Munro CA, Selvaggini S, de Bruijn I, Walker L, Lenardon MD, Gerssen B, et al. The PKC, HOG

677 and Ca2+ signalling pathways co-ordinately regulate chitin synthesis in Candida albicans. Mol Microbiol.

$678 \quad 2007 ; 63(5): 1399-413$.

679 27. Fujioka A, Terai K, Itoh RE, Aoki K, Nakamura T, Kuroda S, et al. Dynamics of the Ras/ERK

680 MAPK cascade as monitored by fluorescent probes. J Biol Chem. 2006;281(13):8917-26. 
681 28. Walker LA, Munro CA, de Bruijn I, Lenardon MD, McKinnon A, Gow NA. Stimulation of chitin

682 synthesis rescues Candida albicans from echinocandins. PLoS Pathog. 2008;4(4):e1000040.

683 29. Rodriguez-Pena JM, Garcia R, Nombela C, Arroyo J. The high-osmolarity glycerol (HOG) and 684 cell wall integrity (CWI) signalling pathways interplay: a yeast dialogue between MAPK routes. Yeast. 685 2010;27(8):495-502.

686 30. Colabardini AC, Ries LN, Brown NA, Savoldi M, Dinamarco TM, von Zeska Kress MR, et al. 687 Protein kinase C overexpression suppresses calcineurin-associated defects in Aspergillus nidulans and is 688 involved in mitochondrial function. PLoS One. 2014;9(8):e104792.

689 31. Garcia R, Bravo E, Diez-Muniz S, Nombela C, Rodriguez-Pena JM, Arroyo J. A novel 690 connection between the Cell Wall Integrity and the PKA pathways regulates cell wall stress response in 691 yeast. Sci Rep. 2017;7(1):5703.

692 32. Manfiolli AO, Mattos EC, de Assis LJ, Silva LP, Ulas M, Brown NA, et al. Aspergillus 693 fumigatus High Osmolarity Glycerol Mitogen Activated Protein Kinases SakA and MpkC Physically 694 Interact During Osmotic and Cell Wall Stresses. Front Microbiol. 2019;10:918.

695 33. Dickson RC. Thematic review series: sphingolipids. New insights into sphingolipid metabolism 696 and function in budding yeast. J Lipid Res. 2008;49(5):909-21.

697 34. Sims KJ, Spassieva SD, Voit EO, Obeid LM. Yeast sphingolipid metabolism: clues and connections. Biochem Cell Biol. 2004;82(1):45-61.

699 35. Roelants FM, Torrance PD, Bezman N, Thorner J. Pkh1 and Pkh2 differentially phosphorylate 700 and activate Ypk1 and Ykr2 and define protein kinase modules required for maintenance of cell wall 701 integrity. Mol Biol Cell. 2002;13(9):3005-28. 
702 36. Weete JD, Abril M, Blackwell M. Phylogenetic distribution of fungal sterols. PLoS One.

$703 \quad$ 2010;5(5):e10899.

704 37. Alvarez FJ, Douglas LM, Konopka JB. Sterol-rich plasma membrane domains in fungi. Eukaryot

705 Cell. 2007;6(5):755-63.

$706 \quad 38 . \quad$ Tanaka S, Tani M. Mannosylinositol phosphorylceramides and ergosterol coodinately maintain

707 cell wall integrity in the yeast Saccharomyces cerevisiae. FEBS J. 2018;285(13):2405-27.

708 39. Bagnat M, Simons K. Lipid rafts in protein sorting and cell polarity in budding yeast

709 Saccharomyces cerevisiae. Biol Chem. 2002;383(10):1475-80.

710 40. van Meer G, Voelker DR, Feigenson GW. Membrane lipids: where they are and how they

711 behave. Nat Rev Mol Cell Biol. 2008;9(2):112-24.

712 41. Tanaka-Takiguchi Y, Kinoshita M, Takiguchi K. Septin-mediated uniform bracing of

713 phospholipid membranes. Curr Biol. 2009;19(2):140-5.

714 42. Bertin A, McMurray MA, Thai L, Garcia G, 3rd, Votin V, Grob P, et al. Phosphatidylinositol-4,5-

715 bisphosphate promotes budding yeast septin filament assembly and organization. J Mol Biol.

$716 \quad 2010 ; 404(4): 711-31$.

717 43. Krokowski S, Lobato-Marquez D, Chastanet A, Pereira PM, Angelis D, Galea D, et al. Septins

718 Recognize and Entrap Dividing Bacterial Cells for Delivery to Lysosomes. Cell Host Microbe.

719 2018;24(6):866-74 e4.

720 44. Zhang J, Kong C, Xie H, McPherson PS, Grinstein S, Trimble WS. Phosphatidylinositol

721 polyphosphate binding to the mammalian septin H5 is modulated by GTP. Curr Biol. 1999;9(24):1458-

72267. 
723 45. Costanzo M, Baryshnikova A, Bellay J, Kim Y, Spear ED, Sevier CS, et al. The genetic

724 landscape of a cell. Science. 2010;327(5964):425-31.

725 46. Clay L, Caudron F, Denoth-Lippuner A, Boettcher B, Buvelot Frei S, Snapp EL, et al. A

726 sphingolipid-dependent diffusion barrier confines ER stress to the yeast mother cell. Elife.

727 2014;3:e01883.

728 47. Martin SW, Konopka JB. Lipid raft polarization contributes to hyphal growth in Candida

729 albicans. Eukaryot Cell. 2004;3(3):675-84.

730 48. Beh CT, Alfaro G, Duamel G, Sullivan DP, Kersting MC, Dighe S, et al. Yeast oxysterol-binding 731 proteins: sterol transporters or regulators of cell polarization? Mol Cell Biochem. 2009;326(1-2):9-13.

732 49. Canovas D, Perez-Martin J. Sphingolipid biosynthesis is required for polar growth in the

733 dimorphic phytopathogen Ustilago maydis. Fungal Genet Biol. 2009;46(2):190-200.

734 50. Singh P, Ramachandran SK, Zhu J, Kim BC, Biswas D, Ha T, et al. Sphingolipids facilitate age

735 asymmetry of membrane proteins in dividing yeast cells. Mol Biol Cell. 2017;28(20):2712-22.

736 51. Sugiyama S, Tanaka M. Distinct segregation patterns of yeast cell-peripheral proteins uncovered

737 by a method for protein segregatome analysis. Proc Natl Acad Sci U S A. 2019;116(18):8909-18.

738 52. Bridges AA, Gladfelter AS. Septin Form and Function at the Cell Cortex. J Biol Chem.

739 2015;290(28):17173-80.

740 53. Merlini L, Bolognesi A, Juanes MA, Vandermoere F, Courtellemont T, Pascolutti R, et al. Rho1-

741 and Pkc1-dependent phosphorylation of the F-BAR protein Syp1 contributes to septin ring assembly. Mol

742 Biol Cell. 2015;26(18):3245-62. 
54. Badrane H, Nguyen MH, Clancy CJ. Highly Dynamic and Specific Phosphatidylinositol 4,5-

744 Bisphosphate, Septin, and Cell Wall Integrity Pathway Responses Correlate with Caspofungin Activity

745 against Candida albicans. Antimicrob Agents Chemother. 2016;60(6):3591-600.

$74655 . \quad$ de Almeida RFM. A route to understanding yeast cellular envelope - plasma membrane lipids

747 interplaying in cell wall integrity. FEBS J. 2018;285(13):2402-4.

748 56. Wu JQ, Ye Y, Wang N, Pollard TD, Pringle JR. Cooperation between the septins and the

749 actomyosin ring and role of a cell-integrity pathway during cell division in fission yeast. Genetics.

$750 \quad 2010 ; 186(3): 897-915$.

751 57. Zhang Y, Gao T, Shao W, Zheng Z, Zhou M, Chen C. The septins FaCdc3 and FaCdc12 are

752 required for cytokinesis and affect asexual and sexual development, lipid metabolism and virulence in

753 Fusarium asiaticum. Mol Plant Pathol. 2017;18(9):1282-94.

754 58. Kuranda K, Leberre V, Sokol S, Palamarczyk G, Francois J. Investigating the caffeine effects in 755 the yeast Saccharomyces cerevisiae brings new insights into the connection between TOR, PKC and

756 Ras/cAMP signalling pathways. Mol Microbiol. 2006;61(5):1147-66.

757 59. Kovacs Z, Szarka M, Kovacs S, Boczonadi I, Emri T, Abe K, et al. Effect of cell wall integrity

758 stress and RlmA transcription factor on asexual development and autolysis in Aspergillus nidulans.

759 Fungal Genet Biol. 2013;54:1-14.

760 60. Roncero C, Duran A. Effect of Calcofluor white and Congo red on fungal cell wall

761 morphogenesis: in vivo activation of chitin polymerization. J Bacteriol. 1985;163(3):1180-5.

762 61. Pancaldi S, Poli F, Dall'Olio G, Vannini GL. Morphological anomalies induced by Congo red in 763 Aspergillus niger. Arch Microbiol. 1984;137(3):185-7. 
62. Kopecka M, Gabriel M. The influence of congo red on the cell wall and (1----3)-beta-D-glucan microfibril biogenesis in Saccharomyces cerevisiae. Arch Microbiol. 1992;158(2):115-26.

63. Onishi J, Meinz M, Thompson J, Curotto J, Dreikorn S, Rosenbach M, et al. Discovery of novel antifungal (1,3)-beta-D-glucan synthase inhibitors. Antimicrob Agents Chemother. 2000;44(2):368-77.

64. Kojima K, Bahn YS, Heitman J. Calcineurin, Mpk1 and Hog1 MAPK pathways independently control fludioxonil antifungal sensitivity in Cryptococcus neoformans. Microbiology. 2006;152(Pt 3):591-604.

\section{Yun Y, Liu Z, Zhang J, Shim WB, Chen Y, Ma Z. The MAPKK FgMkk1 of Fusarium} graminearum regulates vegetative differentiation, multiple stress response, and virulence via the cell wall integrity and high-osmolarity glycerol signaling pathways. Environ Microbiol. 2014;16(7):2023-37.

66. Dongo A, Bataille-Simoneau N, Campion C, Guillemette T, Hamon B, Iacomi-Vasilescu B, et al. The group III two-component histidine kinase of filamentous fungi is involved in the fungicidal activity of the bacterial polyketide ambruticin. Appl Environ Microbiol. 2009;75(1):127-34.

67. Hohmann S. Osmotic stress signaling and osmoadaptation in yeasts. Microbiol Mol Biol Rev. 2002;66(2):300-72.

68. Garcia-Rodriguez LJ, Trilla JA, Castro C, Valdivieso MH, Duran A, Roncero C. Characterization of the chitin biosynthesis process as a compensatory mechanism in the fks1 mutant of Saccharomyces cerevisiae. FEBS Lett. 2000;478(1-2):84-8.

69. Fortwendel JR, Juvvadi PR, Perfect BZ, Rogg LE, Perfect JR, Steinbach WJ. Transcriptional regulation of chitin synthases by calcineurin controls paradoxical growth of Aspergillus fumigatus in response to caspofungin. Antimicrob Agents Chemother. 2010;54(4):1555-63.

70. Pettolino FA, Walsh C, Fincher GB, Bacic A. Determining the polysaccharide composition of plant cell walls. Nat Protoc. 2012;7(9):1590-607. 
787 71. Yoshimi A, Miyazawa K, Abe K. Cell wall structure and biogenesis in Aspergillus species.

788 Biosci Biotechnol Biochem. 2016;80(9):1700-11.

789 72. Yoshimoto H, Saltsman K, Gasch AP, Li HX, Ogawa N, Botstein D, et al. Genome-wide analysis

790 of gene expression regulated by the calcineurin/Crz1p signaling pathway in Saccharomyces cerevisiae. J

791 Biol Chem. 2002;277(34):31079-88.

792 73. Sio SO, Suehiro T, Sugiura R, Takeuchi M, Mukai H, Kuno T. The role of the regulatory subunit

793 of fission yeast calcineurin for in vivo activity and its relevance to FK506 sensitivity. J Biol Chem.

$794 \quad$ 2005;280(13):12231-8.

795 74. Wang S, Liu X, Qian H, Zhang S, Lu L. Calcineurin and Calcium Channel CchA Coordinate the 796 Salt Stress Response by Regulating Cytoplasmic Ca2+ Homeostasis in Aspergillus nidulans. Appl

797 Environ Microbiol. 2016;82(11):3420-30.

798 75. Harvald EB, Olsen AS, Faergeman NJ. Autophagy in the light of sphingolipid metabolism.

799 Apoptosis. 2015;20(5):658-70.

800 76. Barbet NC, Schneider U, Helliwell SB, Stansfield I, Tuite MF, Hall MN. TOR controls

801 translation initiation and early G1 progression in yeast. Mol Biol Cell. 1996;7(1):25-42.

802 77. Cardenas ME, Cutler NS, Lorenz MC, Di Como CJ, Heitman J. The TOR signaling cascade

803 regulates gene expression in response to nutrients. Genes Dev. 1999;13(24):3271-9.

804 78. Scott PH, Lawrence JC, Jr. Attenuation of mammalian target of rapamycin activity by increased 805 cAMP in 3T3-L1 adipocytes. J Biol Chem. 1998;273(51):34496-501.

806 79. Fitzgibbon GJ, Morozov IY, Jones MG, Caddick MX. Genetic analysis of the TOR pathway in 807 Aspergillus nidulans. Eukaryot Cell. 2005;4(9):1595-8. 

Biol Cell. 2010;21(19):3475-86.

811 81. Butcher FR, Potter VR. Control of the adenosine 3',5'-monophosphate-adenyl cyclase system in

812 the livers of developing rats. Cancer Res. 1972;32(10):2141-7.

813 82. Ni M, Rierson S, Seo JA, Yu JH. The pkaB gene encoding the secondary protein kinase A

814 catalytic subunit has a synthetic lethal interaction with pkaA and plays overlapping and opposite roles in 815 Aspergillus nidulans. Eukaryot Cell. 2005;4(8):1465-76.

816 83. Binder U, Oberparleiter C, Meyer V, Marx F. The antifungal protein PAF interferes with

817 PKC/MPK and cAMP/PKA signalling of Aspergillus nidulans. Mol Microbiol. 2010;75(2):294-307.

818 84. Foderaro JE, Douglas LM, Konopka JB. MCC/Eisosomes Regulate Cell Wall Synthesis and

819 Stress Responses in Fungi. J Fungi (Basel). 2017;3(4).

820 85. Onyewu C, Blankenship JR, Del Poeta M, Heitman J. Ergosterol biosynthesis inhibitors become

821 fungicidal when combined with calcineurin inhibitors against Candida albicans, Candida glabrata, and

822 Candida krusei. Antimicrob Agents Chemother. 2003;47(3):956-64.

823 86. Cheng J, Park TS, Fischl AS, Ye XS. Cell cycle progression and cell polarity require sphingolipid 824 biosynthesis in Aspergillus nidulans. Mol Cell Biol. 2001;21(18):6198-209.

825 87. Li S, Bao D, Yuen G, Harris SD, Calvo AM. basA regulates cell wall organization and 826 asexual/sexual sporulation ratio in Aspergillus nidulans. Genetics. 2007;176(1):243-53.

827 88. Walker EH, Pacold ME, Perisic O, Stephens L, Hawkins PT, Wymann MP, et al. Structural 828 determinants of phosphoinositide 3-kinase inhibition by wortmannin, LY294002, quercetin, myricetin, 829 and staurosporine. Mol Cell. 2000;6(4):909-19. 
831 al. Wortmannin inactivates phosphoinositide 3-kinase by covalent modification of Lys-802, a residue

832 involved in the phosphate transfer reaction. Mol Cell Biol. 1996;16(4):1722-33.

833 90. Santos NC, Ter-Ovanesyan E, Zasadzinski JA, Prieto M, Castanho MA. Filipin-induced lesions

834 in planar phospholipid bilayers imaged by atomic force microscopy. Biophys J. 1998;75(4):1869-73.

835 91. Takeo K. A correlation between mode of growth and regional ultrastructure of the plasma

836 membrane of Schizosaccharomyces pombe as revealed by freeze-fracturing before and after filipin

837 treatment. J Gen Microbiol. 1985;131(2):309-16.

838 92. de Kruijff B, Demel RA. Polyene antibiotic-sterol interactions in membranes of Acholeplasma

839 laidlawii cells and lecithin liposomes. 3. Molecular structure of the polyene antibiotic-cholesterol

840 complexes. Biochim Biophys Acta. 1974;339(1):57-70.

841 93. Bjorkbom A, Rog T, Kaszuba K, Kurita M, Yamaguchi S, Lonnfors M, et al. Effect of

842 sphingomyelin headgroup size on molecular properties and interactions with cholesterol. Biophys J.

843 2010;99(10):3300-8.

844 94. Ramstedt B, Slotte JP. Sphingolipids and the formation of sterol-enriched ordered membrane

845 domains. Biochim Biophys Acta. 2006;1758(12):1945-56.

846 95. Bridges AA, Zhang H, Mehta SB, Occhipinti P, Tani T, Gladfelter AS. Septin assemblies form by

847 diffusion-driven annealing on membranes. Proc Natl Acad Sci U S A. 2014;111(6):2146-51.

848 96. Cho H, Stanzione F, Oak A, Kim GH, Yerneni S, Qi L, et al. Intrinsic Structural Features of the

849 Human IRE1alpha Transmembrane Domain Sense Membrane Lipid Saturation. Cell Rep.

$850 \quad$ 2019;27(1):307-20 e5. 
851 97. Nyholm TK, Ozdirekcan S, Killian JA. How protein transmembrane segments sense the lipid

852 environment. Biochemistry. 2007;46(6):1457-65.

853 98. Valadares NF, d' Muniz Pereira H, Ulian Araujo AP, Garratt RC. Septin structure and filament

854 assembly. Biophys Rev. 2017;9(5):481-500.

855 99. Cannon KS, Woods BL, Crutchley JM, Gladfelter AS. An amphipathic helix enables septins to

856 sense micrometer-scale membrane curvature. J Cell Biol. 2019;218(4):1128-37.

857 100. Valiante V, Macheleidt J, Foge M, Brakhage AA. The Aspergillus fumigatus cell wall integrity

858 signaling pathway: drug target, compensatory pathways, and virulence. Front Microbiol. 2015;6:325.

859 101. Valiante V, Jain R, Heinekamp T, Brakhage AA. The MpkA MAP kinase module regulates cell

860 wall integrity signaling and pyomelanin formation in Aspergillus fumigatus. Fungal Genet Biol.

$861 \quad 2009 ; 46(12): 909-18$.

862 102. Valiante V, Heinekamp T, Jain R, Hartl A, Brakhage AA. The mitogen-activated protein kinase

863 MpkA of Aspergillus fumigatus regulates cell wall signaling and oxidative stress response. Fungal Genet

864 Biol. 2008;45(5):618-27.

865 103. Ram AF, Wolters A, Ten Hoopen R, Klis FM. A new approach for isolating cell wall mutants in

866 Saccharomyces cerevisiae by screening for hypersensitivity to calcofluor white. Yeast. 1994;10(8):1019-

86730.

868 104. Ram AF, Kapteyn JC, Montijn RC, Caro LH, Douwes JE, Baginsky W, et al. Loss of the plasma

869 membrane-bound protein Gas1p in Saccharomyces cerevisiae results in the release of beta1,3-glucan into

870 the medium and induces a compensation mechanism to ensure cell wall integrity. J Bacteriol.

871 1998;180(6):1418-24. 
872 105. Kapteyn JC, Ram AF, Groos EM, Kollar R, Montijn RC, Van Den Ende H, et al. Altered extent

873 of cross-linking of beta1,6-glucosylated mannoproteins to chitin in Saccharomyces cerevisiae mutants

874 with reduced cell wall beta1,3-glucan content. J Bacteriol. 1997;179(20):6279-84.

875 106. Kapteyn JC, Hoyer LL, Hecht JE, Muller WH, Andel A, Verkleij AJ, et al. The cell wall

876 architecture of Candida albicans wild-type cells and cell wall-defective mutants. Mol Microbiol.

877 2000;35(3):601-11.

878 107. Popolo L, Gilardelli D, Bonfante P, Vai M. Increase in chitin as an essential response to defects

879 in assembly of cell wall polymers in the ggp1delta mutant of Saccharomyces cerevisiae. J Bacteriol.

$880 \quad$ 1997;179(2):463-9.

881 108. Dallies N, Francois J, Paquet V. A new method for quantitative determination of polysaccharides

882 in the yeast cell wall. Application to the cell wall defective mutants of Saccharomyces cerevisiae. Yeast.

883 1998;14(14):1297-306.

884 109. Osmond BC, Specht CA, Robbins PW. Chitin synthase III: synthetic lethal mutants and "stress 885 related" chitin synthesis that bypasses the CSD3/CHS6 localization pathway. Proc Natl Acad Sci U S A. 886 1999;96(20):11206-10.

887 110. Lagorce A, Le Berre-Anton V, Aguilar-Uscanga B, Martin-Yken H, Dagkessamanskaia A,

888 Francois J. Involvement of GFA1, which encodes glutamine-fructose-6-phosphate amidotransferase, in

889 the activation of the chitin synthesis pathway in response to cell-wall defects in Saccharomyces

890 cerevisiae. Eur J Biochem. 2002;269(6):1697-707.

891 111. Perez J, Arcones I, Gomez A, Casquero V, Roncero C. Phosphorylation of Bni4 by MAP kinases

892 contributes to septum assembly during yeast cytokinesis. FEMS Yeast Res. 2016;16(6).

893 112. Heinisch JJ, Rodicio R. Protein kinase C in fungi-more than just cell wall integrity. FEMS

894 Microbiol Rev. 2018;42(1). 
895 113. Larson JR, Kozubowski L, Tatchell K. Changes in Bni4 localization induced by cell stress in

896 Saccharomyces cerevisiae. J Cell Sci. 2010;123(Pt 7):1050-9.

897 114. Harris SD, Morrell JL, Hamer JE. Identification and characterization of Aspergillus nidulans

898 mutants defective in cytokinesis. Genetics. 1994;136(2):517-32.

899 115. Momany M, Westfall PJ, Abramowsky G. Aspergillus nidulans swo mutants show defects in

900 polarity establishment, polarity maintenance and hyphal morphogenesis. Genetics. 1999;151(2):557-67. 
A.
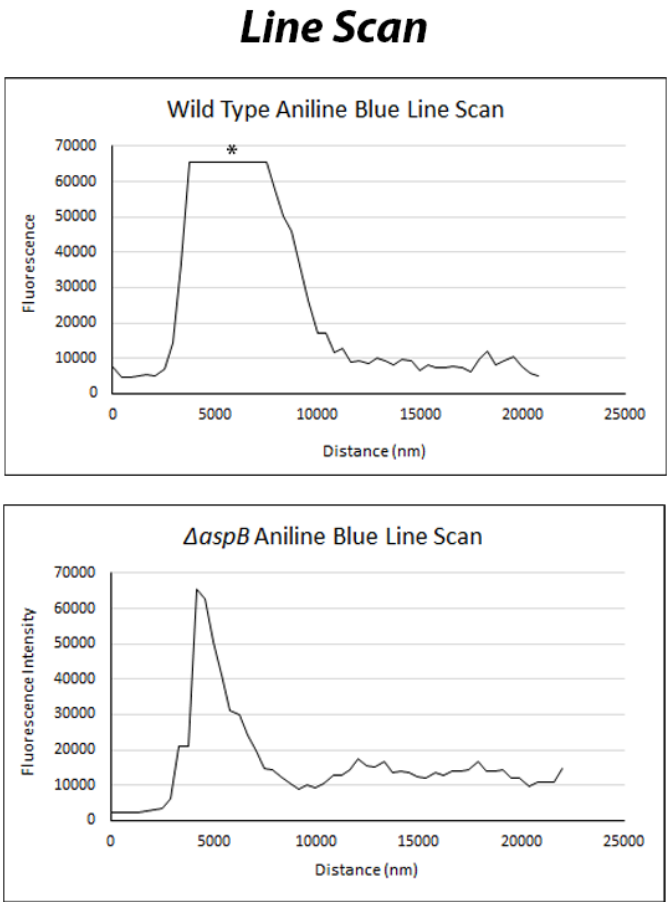

B.
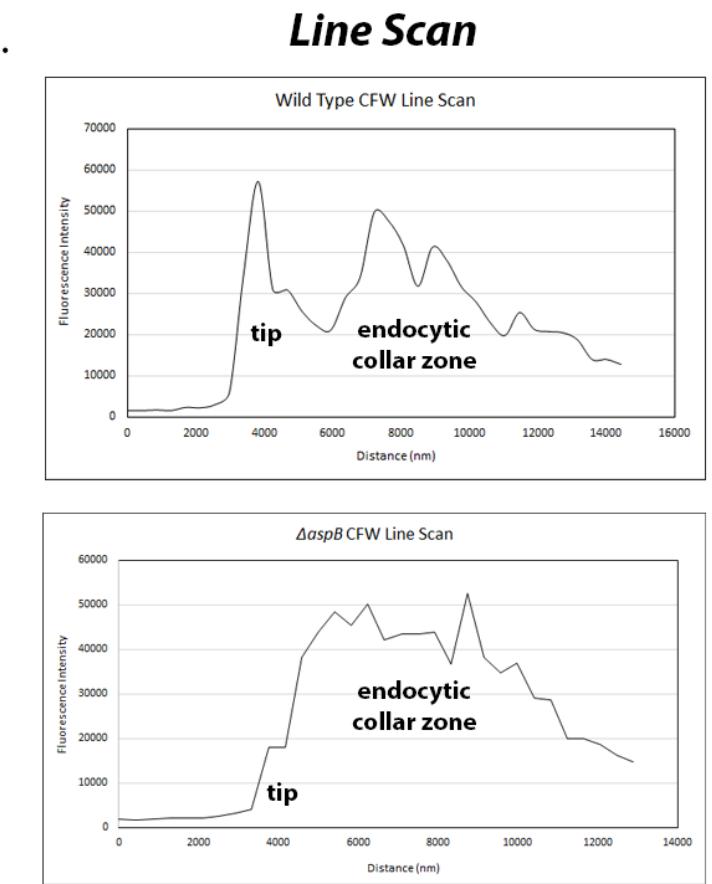

Aniline Blue

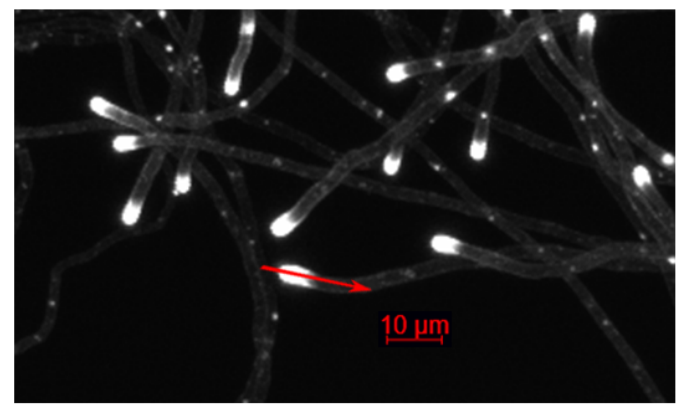

WT

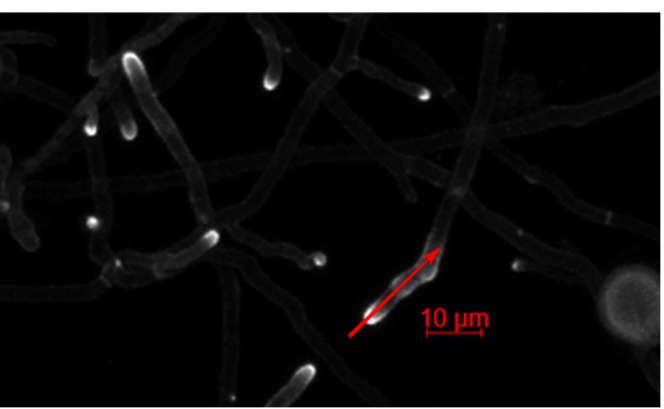

$\triangle a s p B$

WT
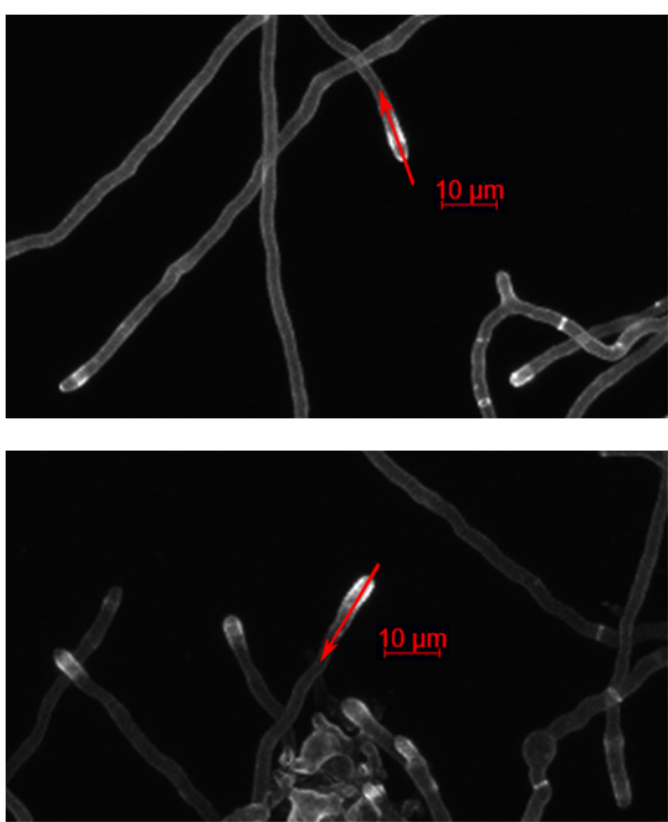

$\triangle a s p B$

S1 Fig. Line scans of cell wall staining reveals unique patterns of chitin and $\beta$-1,3-glucan deposition 


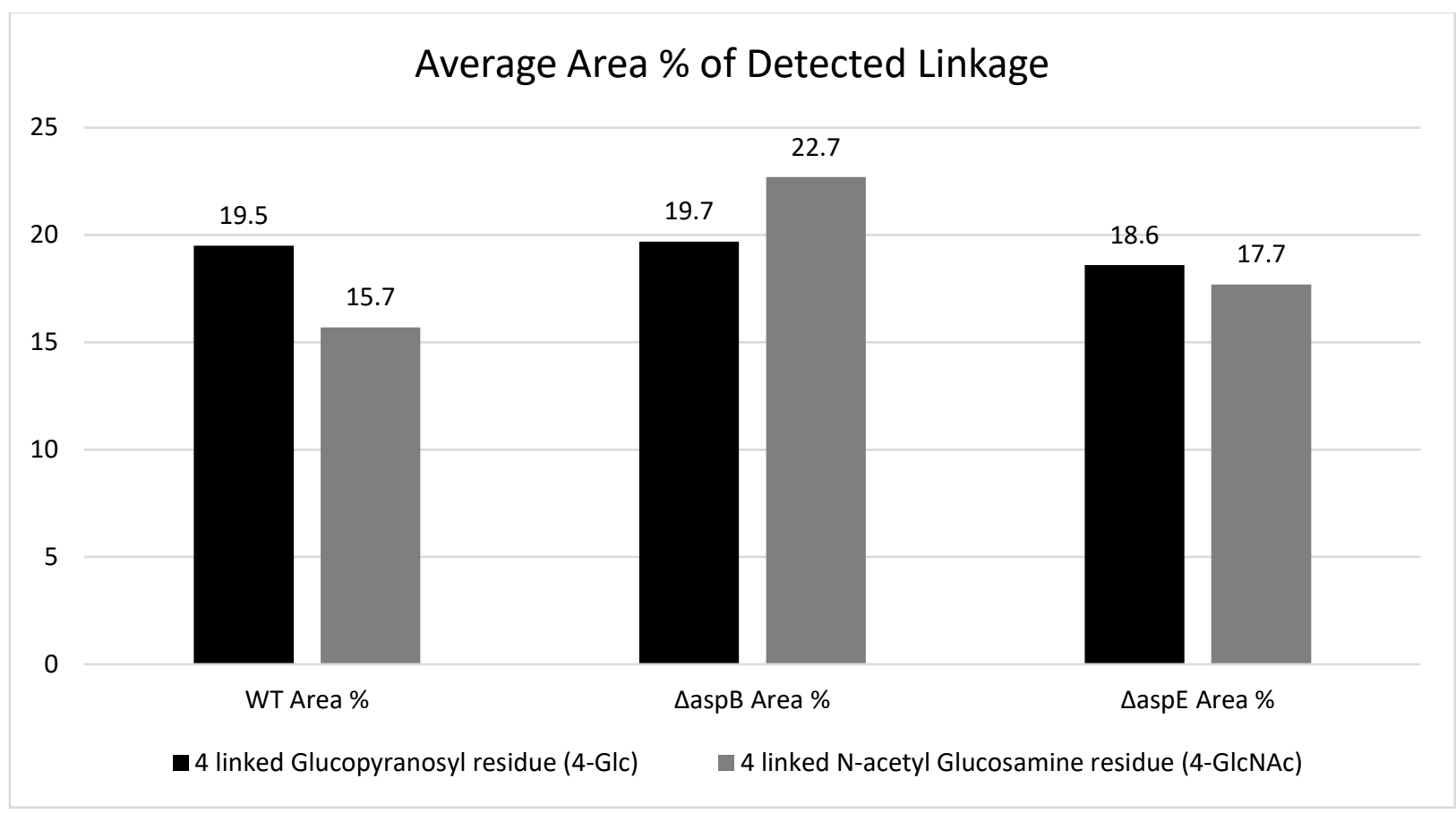

917 S2 Fig. Cell wall glycosyl linkage analysis shows increased chitin content in $\triangle a s p B^{c d c 3}$ septin null

918 mutant. Results of cell wall polysaccharide glycosyl linkage analysis using GC MS/MS showing the 919 average area (\%) of detected linkages of 4-linked glucose and 4-linked $\mathrm{N}$-acetyl glucosamine. Two

920 independent biological replicates gave similar results. A representative data set is shown. Samples: WT, $921 \Delta a s p B^{c d c 3}$, and $\triangle a s p E$. 


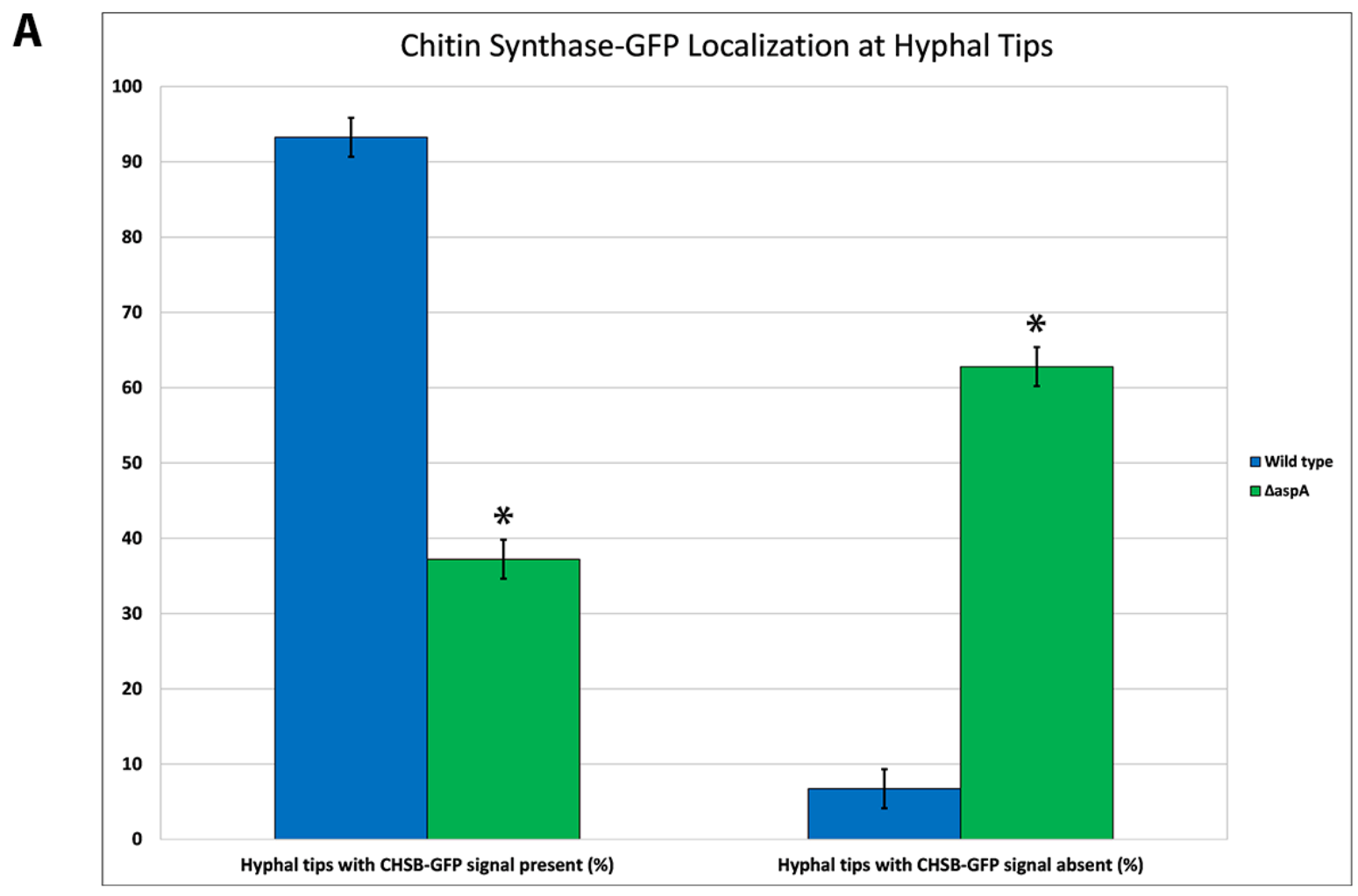

B

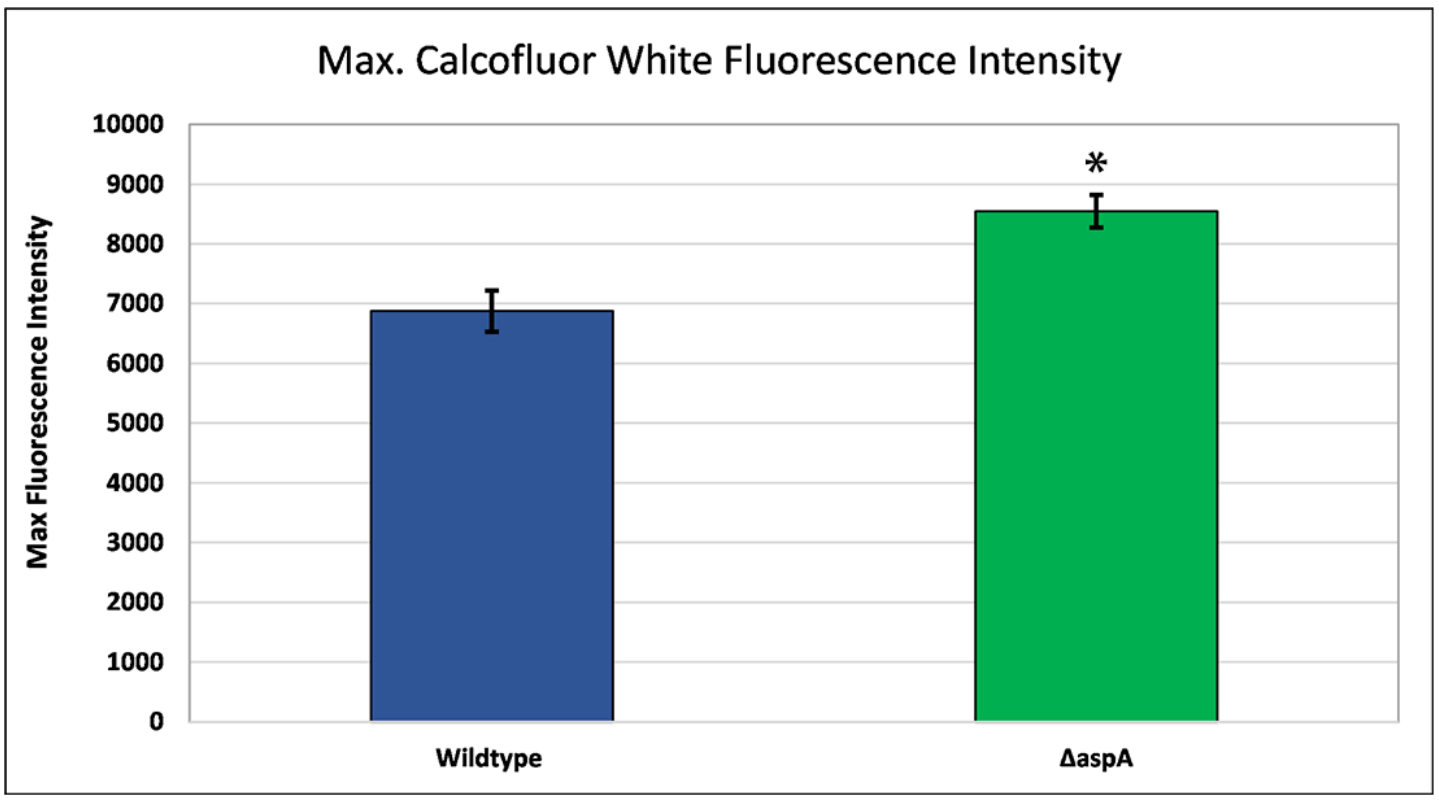
percentage (\%) of hyphal branch tips with chitin synthase B-GFP present vs. absent by line scans in WT

927 and $\Delta a s p A^{c d c 11}$. Mean values shown for line scans of $\geq 85$ hyphal branches over two independent

928 biological replicates. Asterisks indicate different standard error of the mean between sample sets. (B)

929 Quantification of maximum fluorescence intensity by line scans of CFW stained WT and $\Delta a s p A^{c d c 11}$ 

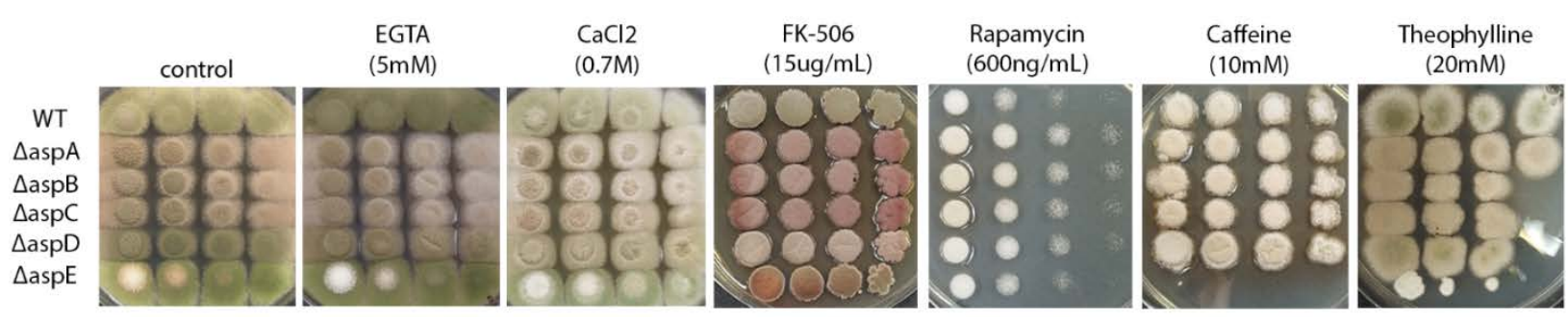

S4 Fig. Core septin null mutants are not sensitive to treatments which disrupt the Ca2+/Calcineurin or TOR pathways. AspE is hypersensitive to treatments which disrupt the TOR pathway. WT and septin null mutants $\Delta a s p A^{c d c 11}, \Delta a s p B^{c d c 3}, \Delta a s p C^{c d c 12}, \Delta a s p D^{c d c 10}$, and $\Delta a s p E$ were tested for sensitivity by spotting decreasing spore concentrations on complete media plates with or without Calcium/Calcineurin pathway-disturbing agents (EGTA, CaCl2, and FK-506) or Target of Rapamycin (TOR) pathwaydisturbing agents (Rapamycin, caffeine, and theophylline). Differences in colony color result from
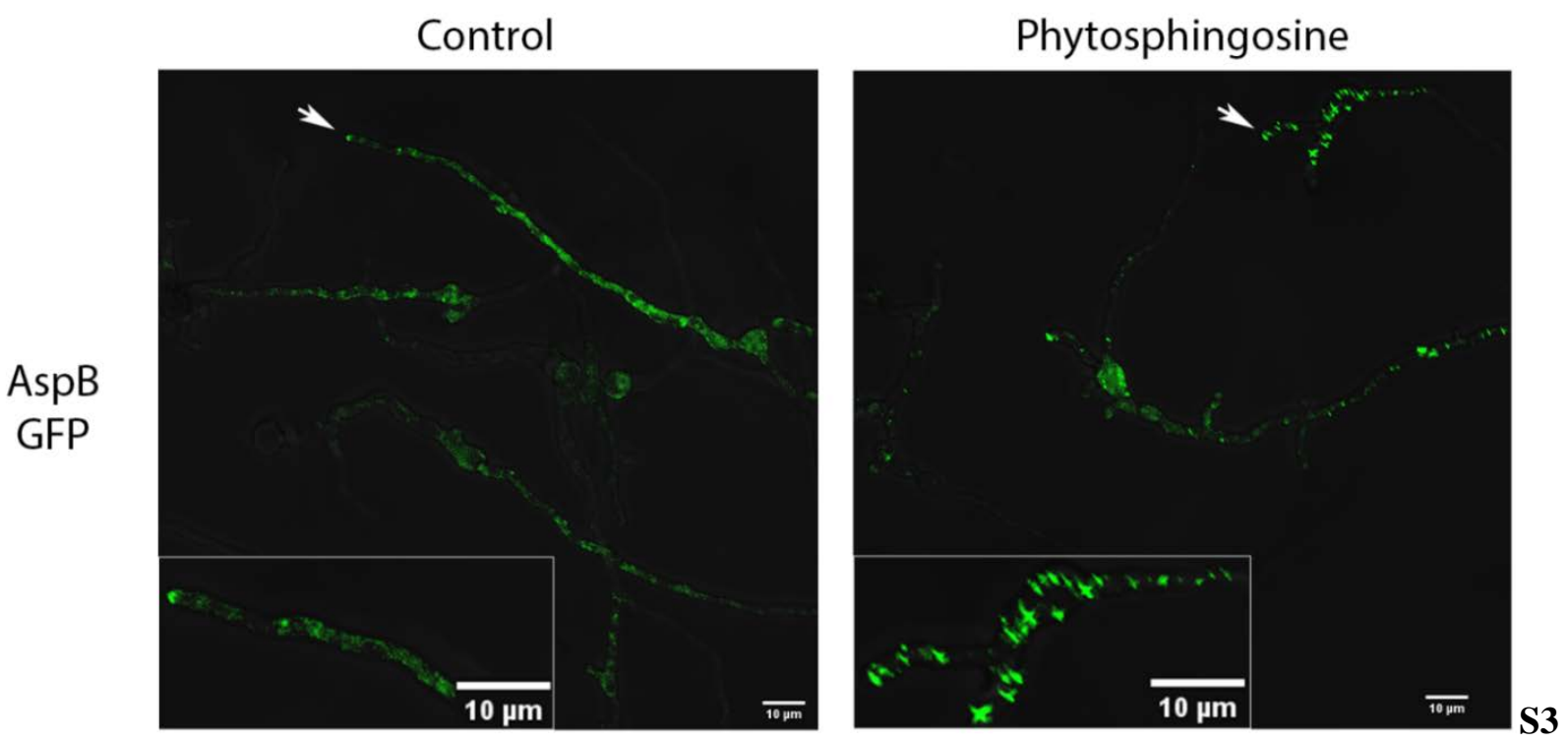

S5 Fig. Septin AspB ${ }^{\mathrm{Cdc} 3}$-GFP localization disrupted by sphingolipid biosynthesis intermediate phytosphingosine. AspB ${ }^{\mathrm{Cdc} 3}$-GFP strain incubated in liquid media for approximately $16 \mathrm{~h}$ and imaged 180 minutes after replacing with fresh media containing sphingolipid biosynthesis-inhibiting agents. 
Representative images are shown from three independent biological replicates, with $\geq 100$ cells observed. (Left Panel) AspB-GFP in vehicle control treatments and (Right Panel) phytosphingosine (15 $\mu \mathrm{M})$ treatment. Enlarged section of micrographs from each picture to better visualize pattern of fluorescence. White arrows denote hyphae which are highlighted in enlarged images. Imaging conducted with Deltavision I deconvolution inverted fluorescence microscope. Scale bars $=10 \mu \mathrm{m}$. N=3
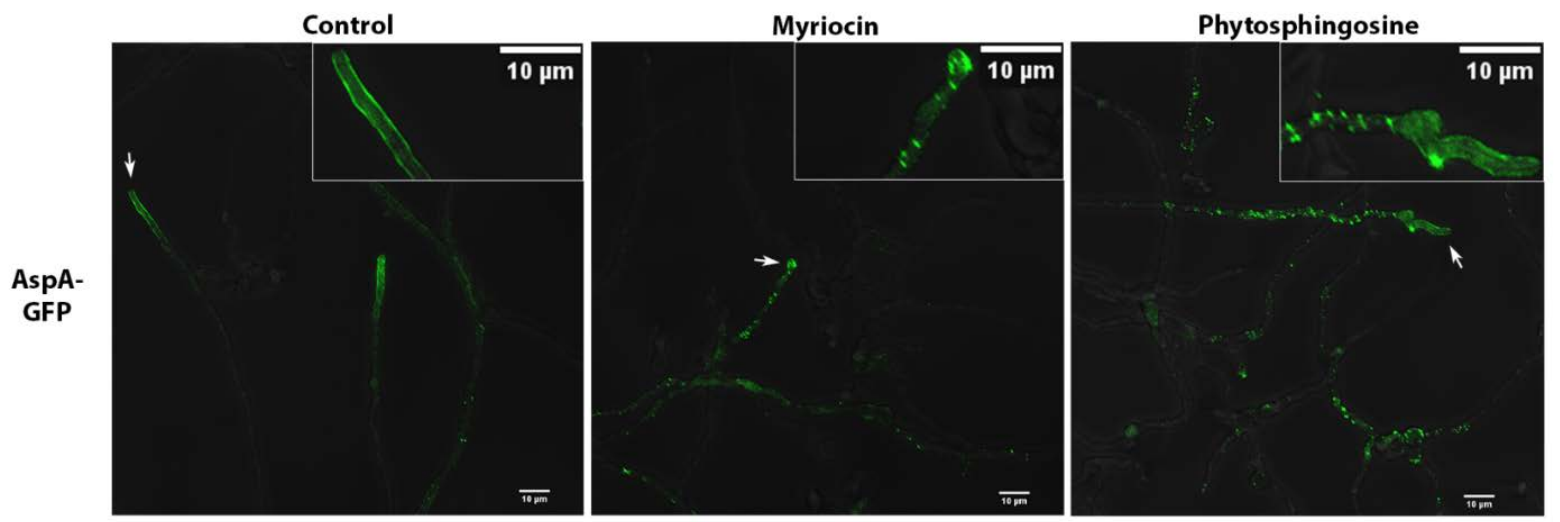

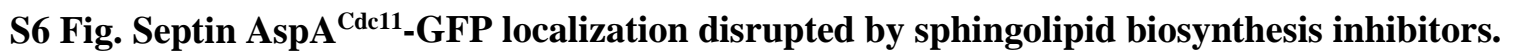

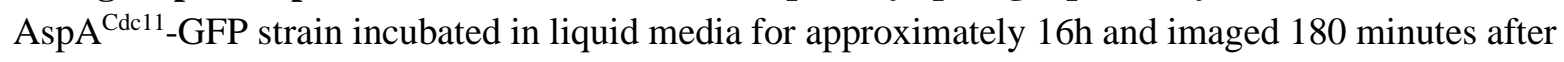

960 replacing with fresh media containing sphingolipid biosynthesis-inhibiting agents. Representative images 961 are shown from three independent biological replicates, with $\geq 100$ cells observed. (Left Panel) AspA-

962 GFP in vehicle control treatment, (Middle Panel) Myriocin $(17.5 \mu \mathrm{g} / \mathrm{mL}$ ) and (Right Panel)

963 phytosphingosine $(15 \mu \mathrm{M})$ treatment. Enlarged section of micrographs from each picture to better

964 visualize pattern of fluorescence signal. White arrows denote hyphae which are highlighted in enlarged 965 images. Imaging conducted with Deltavision I deconvolution inverted fluorescence microscope. Scale 966 bars $=10 \mu \mathrm{m} . \mathrm{N}=3$ 
971 S1 Table. List of fungal strains used in this study.

\begin{tabular}{|c|c|c|}
\hline Strain Number & Genotype & Source \\
\hline FGSC A850 (WT) & biA1; _argB::trpC_B; methG1; veA1; trpC801 & FGSC \\
\hline ASH5 $(\Delta a s p A)$ & aspA::argB2 biA1 argB::trpC_B methG1 veA1 trpC801 & $\begin{array}{l}\text { Lindsey and } \\
\text { Momany, } 2010\end{array}$ \\
\hline AYR32 ( $\triangle a s p B)$ & aspB::AfpyrG; pyroA4; argB2 & $\begin{array}{l}\text { Hernandez- } \\
\text { Rodriguez et al., } \\
2012\end{array}$ \\
\hline ARL161 ( $(a s p C)$ & $\begin{array}{l}\text { aspC::AfpyrG pyrG89 biA1 argB::trpC_B methG1 veA1 } \\
\text { trpC801 }\end{array}$ & $\begin{array}{l}\text { Lindsey and } \\
\text { Momany, } 2010\end{array}$ \\
\hline AKK3 $(\Delta a s p D)$ & aspD::AfpyrG; pyroA4; argB2 & $\begin{array}{l}\text { Hernandez- } \\
\text { Rodriguez et al., } \\
2014\end{array}$ \\
\hline ASH41 ( $\triangle a s p E)$ & aspE::AfpyrG; riboB2 & $\begin{array}{l}\text { Hernandez- } \\
\text { Rodriguez et al., } \\
2012\end{array}$ \\
\hline $\begin{array}{l}\text { ANID_05666 } \\
(\Delta \mathrm{mpkA})\end{array}$ & 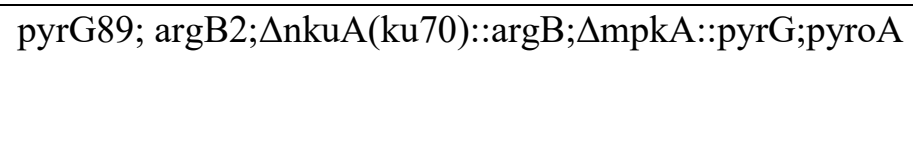 & $\begin{array}{l}\text { CP De Souza et } \\
\text { al., } 2013\end{array}$ \\
\hline $\begin{array}{l}\text { AAM016 } \\
(\Delta \mathrm{mpkA} \triangle \mathrm{aspB})\end{array}$ & 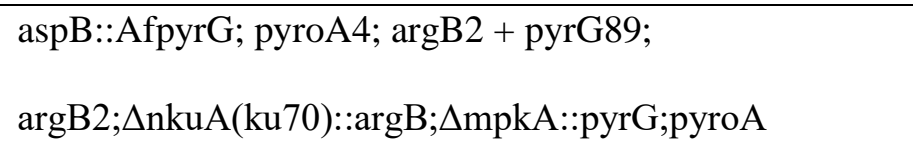 & This study \\
\hline $\begin{array}{l}\text { AAM017 } \\
(\Delta \mathrm{mpkA} \triangle \operatorname{aspB})\end{array}$ & 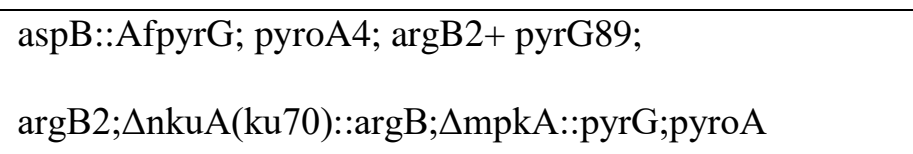 & This study \\
\hline
\end{tabular}




\begin{tabular}{|c|c|c|}
\hline $\begin{array}{l}\text { AAM019 } \\
(\triangle \mathrm{mpkA} \triangle \operatorname{aspE})\end{array}$ & $\begin{array}{l}\text { aspE::AfpyrG; riboB2+ } \\
\text { pyrG89;argB2; } \Delta \text { nkuA(ku70)::argB; } \Delta \mathrm{mpkA}:: p y r G ; p y r o A\end{array}$ & This study \\
\hline $\begin{array}{l}\text { AAM020 } \\
(\triangle \mathrm{mpkA} \triangle \mathrm{aspE})\end{array}$ & 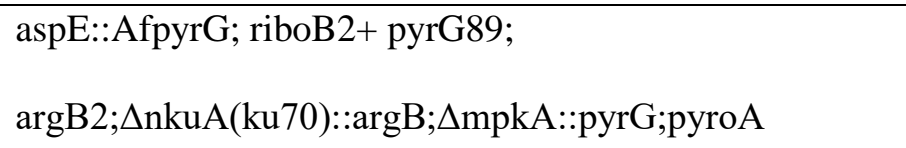 & This study \\
\hline ARL141 & $\begin{array}{l}\text { aspA-GFP-AfpyrG pyrG89 biA1 argB::trpC_B methG1 } \\
\text { veA1 trpC801 }\end{array}$ & $\begin{array}{l}\text { Lindsey and } \\
\text { Momany, } 2010\end{array}$ \\
\hline EB-5 & $\begin{array}{l}\text { biA1 pyrG89 argB2 pyroA4 wA3 } \triangle \text { chsB::pyr-4-alcA(p)- } \\
\text { chsB argB::chsB(p)-egfp-chsB }\end{array}$ & $\begin{array}{l}\text { Fukuda et al., } \\
2009\end{array}$ \\
\hline AAM022 & $\begin{array}{l}\operatorname{asp} A: \because \arg B 2 \text { biA1 pyrG89 argB2 wA3 } \triangle \text { chsB }: \text { pyr-4- } \\
\text { alcA(p)-chsB argB::chsB(p)-egfp-chsB }\end{array}$ & This study \\
\hline
\end{tabular}

973 S2 Table. List of primers and sequences used in this study.

\begin{tabular}{|c|c|}
\hline Primer Name & Primer Sequence \\
\hline PyrG-Af-R' & 5’-CAG AGC CCA CAG AGC GCC TTG AG-3’ \\
\hline AspB-KO-F’ & 5’-GGT CAT TCC TGG TGT GAC AGT ACC-3’ \\
\hline AspE-KO-F' & 5’-GAT CCA AAT TCC AGG TTC GAT GAC-3’ \\
\hline MpkA-806-F' & 5’-ATC CTA GAC TCG ACG CCT CA-3’ \\
\hline MpkA-3779-R' & 5’-ACA AAA ACC CCA TCG TCC GA-3' \\
\hline AspA-KO-F' & 5’-TAG ATC AAG CTC CGC CGG AA-3’ \\
\hline AspA-KO-R' & 5’-TGA CTC CAG CGA CGA TGA GT-3’ \\
\hline
\end{tabular}

